\title{
Stark Ladder Resonances for Small Electric Fields
}

\section{J.-M. Combes ${ }^{\star}$ and P. D. Hislop ${ }^{\star, \star \star \star \star \star \star \star}$}

Centre de Physique Théorique, CNRS, Luminy Case 907, F-13288 Marseille Cedex 9, France

Received October 15, 1990

\begin{abstract}
We prove the existence of resonances in the semi-classical regime of small $h$ for Stark ladder Hamiltonians $H(h, F) \equiv-h^{2} \frac{d^{2}}{d x^{2}}+v+F x$ in onedimension. The potential $v$ is a real periodic function with period $\tau$ which is the restriction to $\mathbb{R}$ of a function analytic in a strip about $\mathbb{R}$. The electric field strength $F$ satisfies the bounds $\left\|v^{\prime}\right\|_{\infty}>F>0$. In general, the imaginary part of the resonances are bounded above by $c e^{-\kappa \rho_{T} h^{-1}}$, for some $0<\kappa \leqq 1$, where $\rho_{T} h^{-1}$ is the single barrier tunneling distance in the Agmon metric for $v+F x$. In the regime where the distance between resonant wells is $\mathcal{O}\left(F^{-1}\right)$, we prove that there is at least one resonance whose width is bounded above by $c e^{-\alpha / F}$, for some $\alpha, c>0$ independent of $h$ and $F$ for $h$ sufficiently small. This is an extension of the Oppenheimer formula for the Stark effect to the case of periodic potentials.
\end{abstract}

\section{Introduction}

The Hamiltonian for an electron moving under the influence of a periodic potential and a constant electric field of strength $F \geqq 0$ in one-dimension is

$$
H(h, F)=-h^{2} \frac{d^{2}}{d x^{2}}+v+F x .
$$

The real periodic potential $v$ is assumed to be the restriction to $\mathbb{R}$ of a function analytic in a strip about the real axis. We consider the small electric field regime $\left\|v^{\prime}\right\|_{\infty}>F>0$ in the semi-classical limit. We prove under these conditions that

\footnotetext{
* Also PHYMAT, Départment de Mathématiques, Université de Toulon et du Var, F-83130 La Garde, France

$\star \star$ Permanent address: Mathematics Department, University of Kentucky, Lexington, KY 40506-0027, USA

$\star \star \star$ Partially supported by NSF Grant DMS- 8911242
} 
there exists $h_{0}>0$ such that for $0<h<h_{0}, H(h, F)$ has infinitely-many spectral resonances, the so-called Stark ladder resonances.

We also prove an upper bound on the width of these resonances. The following bound is an immediate consequence of our work and standard semiclassical analysis of tunneling (see [CDKS, HiSi or Si1]). Let $z_{n, k}$ be any resonance and let $e_{n, k} \equiv \operatorname{Re} z_{n, k}$. Let $h^{-1} \rho_{T}$ be the exit barrier tunneling distance in the Agmon metric for $v(x)+F x$ at energy $e_{n, k}$, where

$$
\rho_{T}=\int_{S_{T}\left(e_{n, k}\right)}^{s_{T}^{\prime}\left(e_{n, k}\right)}\left(v(s)+F s-e_{n, k}\right)_{+}^{1 / 2} d s,
$$

$S_{T}\left(e_{n, k}\right) \equiv \min \left\{x \mid v(x)+F x=e_{n, k}\right\}$ and $S_{T}^{\prime}\left(e_{n, k}\right)$ is the next such point to the right. Then there exist $c_{n, k}>0$ and $0<\beta \leqq 1$ such that

$$
\left|\operatorname{Im} z_{n, k}\right| \leqq c_{n, k} e^{-\beta \rho T / h}
$$

We provis here a more interesting result which explicitly indicates the dependence of $\operatorname{Im} z_{n, l}$ on the electric field strength $F$. Under the condition that resonant wells at energy $e_{0}$ are sufficiently far apart, we prove that there exists a resonance with $\operatorname{Re} z_{0, k}=e_{0}+k F \tau, \tau$ the period of $v$ and $e_{0}$ the ground state of a single cell Hamiltonian, for which there exist constants $c, \alpha>0$ such that

$$
\left|\operatorname{Im} z_{0, k}\right| \leqq c e^{-\alpha / F}
$$

for $h$ sufficiently small. The bound (1.4) can be considered as an extension to periodic potentials of the Oppenheimer formula for the lifetime of a state of the hydrogen atom in a constant electric field $F$ (cf. [H]). This dependency of the width on $F$ was conjectured by Avron [A1].

The results of this paper were announced in [CH1] and in the 1989 Brasov Conference Proceedings [CH2].

Our analysis of Stark ladder resonances follows the general lines of study for shape and Stark resonances. It is based on spectral deformation methods, multi-well tunneling effects, and non-trapping properties of the potential in the semi-classical $($ small $h$ ) regime. Shape resonances in the semi-classical regime were studied by Combes, Duclos, Klein, Seiler [CDKS], Hislop and Sigal [HiSi], Helffer and Sjöstrand [HSj1], Sigal [Si1], and Briet, Combes, Duclos [BCD4]. Semi-classical multi-well tunneling phenomena has been extensively explored in recent years and we mention the work of Combes, Duclos, Seiler [CDS] in one-dimension and the work of Briet, Combes, Duclos [BCD3], Simon [S1,S2] and the exhaustive analysis of Helffer and Sjöstrand [HSj2-4] all for the multidimensional case. Non-trapping estimates on resolvents play a key role in the proof of the existence of spectral resonances in Stark and shape resonance problems. Originally developed for complex exterior scaling ([K] and [BCD1]), general quantum non-trapping conditions and related theorems concerning bounds on resolvents appear in [Si1, DeBH, N, BCD4].

We mention two features of the Stark ladder problem which distinguish it from the shape resonance and atomic Stark problems. First, the electron always experiences the periodic potential $v$. So, despite the force of the constant electric field, it is a priori unclear that the electron moves off to minus infinity. That the electron does eventually escape to minus infinity is the content of the non-trapping 
condition proved in Sect. 3. Second, it has been discussed (cf. [A1] and references therein) that for small electric field strength $F$, the lifetime of Stark ladder resonances is a sensitive function of $F$. Using the ideas of multi-well tunneling, our analysis verifies this result (see Sect. 7). These fluctuations in the lifetime are due to the fact that various potential wells become resonant or non-resonant as $F$ varies and this changes the tunneling probability and hence the lifetime.

There is a large physics literature concerning Stark ladder resonances and we refer to [A1] for additional references. We mention in particular the work of Avron [A1] on the width of these resonances. He discusses the sensitivity of the lifetime to small changes in $F$ and presents physical arguments for some of the results of this paper. In an earlier paper, Avron and Zak [AZ] discuss the spectral concentration for the Hamiltonian (1.1). They show in a solvable, single band model that the spectral density

$$
P(E) \equiv-\pi^{-1} \operatorname{Im}(H(F)-E-i 0)^{-1}
$$

is a continuous function of $F$ concentrated in the band. This indicates a type of "stability of bands" for $F>0$. Indeed, if the interband matrix elements of $H(h, F)$ are neglected, the spectrum of $H(h, F)$ in each band subspace is pure point [A2] (these eigenvalues correspond to the real parts of resonances). We also mention the numerical work of Bentosela, Grecchi, and Zironi [BGZ] who compute the lifetime of resonances for a semi-infinite lattice with a Kronig-Penny potential. Their results are in agreement with the conjectures of Avron [A1] and results of this work.

There are fewer rigorous results on Stark ladder resonances. Agler and Froese [AF] gave the first proof of the existence of Stark ladder resonances for large $F$ (and not in the semi-classical region). Their proof is based on a Birman-Schwinger principle and is quite different from our work. We mention that when $F>\left\|v^{\prime}\right\|_{\infty}$, the potential wells disappear and our analysis no longer holds whereas [AF] establishes the existence of resonances in this case. These must be located far in the lower-half complex plane. Bentosela [B] obtained estimates on the long-time evolution of $e^{i t H(h, F)} \psi$ which suggest that the resonance width is at most linear in $F$ for small $F$. Nenciu and Nenciu [NN] pursued the analysis based on the fact that $H(h, F)$ restricted to the $(F=0)$ band subspace has pure point spectrum and studied the perturbation given by the interband matrix elements.

During the course of our work, two other groups obtained results on (1.1) in different regimes and using different methods. Buslaev and Dimitrieva [BD 1-2] studied the asymptotics of (1.1) with $h=1$ as $F \rightarrow 0$. They obtain the existence of ladders of resonances and an asymptotic expansion in $F$ for the resonance width whose leading coefficient is $e^{-\alpha / F}$ for some $\alpha>0$, which is explicit. Bentosela and Grecchi [BG] have studied another slight variant of (1.1) in which they set $h=\varepsilon h_{0}$, $F=\varepsilon F_{0}$, and consider $\varepsilon \rightarrow 0$ with $h_{0}>0$ fixed and $F_{0}>0$ fixed but sufficiently small. In this limit, they obtain results on the existence and width of the resonance similar to ours. Their approach gives rigorous justification of the tilted band picture of Zener.

This paper is organized as follows. In Sect. 2, we describe the model and an approximate Hamiltonian $H_{0}(h, F)$. The Hamiltonian $H(h, F)$ is considered to be a perturbation of this operator. The non-trapping property of the potential $V=F x+v$ is proved in Sect. 3. This requires the construction of a particular 
vector field $f$ with support in the region where a classical particle with energy $e_{0}$ behaves like a free particle. Analytic families of operators $H_{1}(h, F)$ and $H_{0, t}(h, F)$ are constructed in Sect. 4 using this vector field $f$ and standard spectral deformation methods (see $[\mathrm{Hz}]$ ). In Sect. 5 we prove a crucial resolvent estimate for $H_{0, t}(h, F)$, $\operatorname{Im} t>0$, using the non-trapping result of Sect. 3. The existence of the Stark ladder resonance is proved in Sect. 6 and the crude upper bound (1.2) on the resonance width is given. Section 7 is devoted to proving the refined estimate (1.3) in the case when the resonant wells are separated by a Euclidean distance $\mathcal{O}\left(F^{-1}\right)$. Finally, we conclude with the two appendices. In the first, we sketch the proof of a decay result on a localized resolvent based on [BCD3]. In the second, we discuss a factorization trick (see also [BCD3]), which simplifies tunneling analysis.

\section{The Model and Approximate Hamiltonian}

We consider the Hamiltonian $H(h, F)$ in (1.1) with $h>0$ and $F \geqq 0$ satisfying $\left\|v^{\prime}\right\|_{\infty}>F \geqq 0$. We write $V \equiv v+F x$ for the full potential. We will occasionally omit writing $h$ and $F$ for convenience. Also, we let $c$ denote a generic constant (independent of $h$ ) whose value may change from line to line. We assume the following condition on $v$ :

(v1) $v$, a real-valued non-constant periodic function with period $\tau$, is the restriction to $\mathbb{R}$ of a function $v(z)$ analytic in a strip $S_{\eta}=\{z \in \mathbb{C}|| \operatorname{Im} z \mid<\eta\}$ for some $\eta>0$.

For $F>0$ and any $e$, let $S_{T}(e) \equiv \min \{x \mid F x+v(x)=e\}$, the classical turning point for energy $e$. Note that $V^{\prime}\left(S_{T}(e)\right) \geqq 0$. We distinguish two cases: the threshold case $(\mathrm{TH})$ when $V^{\prime}\left(S_{T}(e)\right)=0$, and the non-threshold case $(\mathrm{NTH})$ when $V^{\prime}\left(S_{T}(e)\right)>0$. We will only consider the NTH case in this paper. (Of course, by varying $F$ slightly, this can always be achieved.) Let $v_{0} \equiv \max _{x} v(x)$. For any $e$, let $G(e) \equiv\{x \mid V(x)-e>0\}$, the classically forbidden region for energy $e$.

It follows by an application of the theorem of Lavine and Faris [RS1] that $H(h, F)$ is essentially self-adjoint on $C_{0}^{\infty}(\mathbb{R})$ for $F \geqq 0$. The following lemma summarizes the spectral properties of $H(h, F)$ for $F \geqq 0$.

Lemma 2.1.

(i) $F=0, \sigma(H(h, 0))=\sigma_{a c}=\bigcup_{n=1}^{\infty} B_{n}$, where $B_{n}=\left\{\alpha_{n}, \beta_{n}\right\}$ is the $n^{\text {th }}$ band and $\alpha_{1}<\beta_{1} \leqq \alpha_{2}<\beta_{2} \leqq \cdots$ and $\alpha_{1} \geqq \max _{x} v(x)$.

(ii) $F>0, \sigma(H(h, F))=\sigma_{a c}=\mathbb{R}$.

Proof. Results (i) is standard; cf. [RS2]. For $F \neq 0$, this is a result of [BCDSSW]: $\sigma_{s c}(H)=\varnothing$ is proved using Mourre theory [CFKS] with conjugate operator $A \equiv \frac{d}{d x}$ and a clever compactness argument; $\sigma_{p p}(H(h, F))=\varnothing$ follows from a theorem in ODE's; $e_{\text {ess }}(H(h, F))=\mathbb{R}$ is demonstrated by constructing a Weyl sequence by the WKB method for each $\lambda \in \mathbb{R}$.

We now introduce our approximate Hamiltonian $H_{0}(h, F)$. We will always consider $F>0$ fixed and $F<\left\|v^{\prime}\right\|_{\infty}$. Let $V_{0} \equiv V \mid[s, s+\tau)$, where $s$ is any value 
such that $v(s)=v_{0}$. By a unit cell of $V$, we mean any interval of the form $[s, s+\tau]$. We extend $V_{0}$ in a continuous, monotonically increasing manner to a potential $\widetilde{V}_{0}$ on $\mathbb{R}$ such that $\lim _{|x| \rightarrow \infty} \widetilde{V}_{0}(x)=+\infty$ and $\widetilde{V}_{0}(x)=\mathcal{O}(|x|)$. Let $\widetilde{h}_{0} \equiv h^{2} p^{2}+\widetilde{V}_{0}$ on $L^{2}(\mathbb{R}), p \equiv-i \frac{d}{d x}$, so $\sigma\left(\tilde{h}_{0}\right)$ is discrete and all multiplicities are one. The operator $\tilde{h}_{0}$ is a single-cell Hamiltonian. Let $\tilde{e}_{0}$ be the lowest eigenvalue of $\tilde{h}_{0}$. In the semi-classical regime of small $h, \tilde{e}_{0}$ is insensitive to the extension of $V_{0}$.

We define $S_{T}\left(\tilde{e}_{0}\right)$, the classical turning point for particles with energy $\tilde{e}_{0}$, by

$$
S_{T}\left(\tilde{e}_{0}\right) \equiv \min \left\{x \mid F x+v(x)=\tilde{e}_{0}\right\}
$$

for the NTH case. We fix the energy scale and the $x$-coordinate by choosing (via a linear change of coordinates) $x=0$ to be $s+\tau$, where $s$ is any point satisfying $v(s)=v_{0}$ as above; then $V(0)=v(0)=v_{0}$. The number of unit cells between $x=0$ and $x=S_{T}\left(\tilde{e}_{0}\right)<0$ is given by

$$
N \equiv\left[\left(v_{0}-\tilde{e}_{0}\right)(F \tau)^{-1}\right],
$$

where $[a]$ is the largest integer less than $a$.

By the continuity of $V$, for any $\varepsilon_{1}>0$ sufficiently small, we can choose $0<\eta_{1}<\eta_{2}$ sufficiently small such that $\left(V-\tilde{e}_{0}\right) \mid\left[S_{T}+\eta_{1}, S_{T}+\eta_{2}\right]>\varepsilon_{1}$ (more conditions on $\eta_{i}$ will be given below). Let $W_{1} \equiv\left(-\infty, S_{T}\left(\tilde{e}_{0}\right)+\eta_{2}\right], W_{2} \equiv$ $\left[S_{T}\left(\tilde{e}_{0}\right)+\eta_{1}, \infty\right)$ and $\Omega \equiv W_{1} \cap W_{2}$. (See Fig. 2.1.) We define a symmetric operator $\tilde{H}_{1}(h, F)$ on $L^{2}\left(W_{1}\right)$ with domain $C_{0}^{\infty}\left(W_{1}\right)$ by

$$
\left(\tilde{H}_{1}(h, F) g\right)(x)=\left(h^{2} p^{2}+F x+v(x)\right) g(x) .
$$

It is easy to check that $\tilde{H}_{1}$ is limit point at $-\infty$ and limit circle at $S_{T}+\eta_{2}$ and hence has defect indices equal to one. We define $H_{1}(h, F)$ to be the self-adjoint extension of $\widetilde{H}_{1}$ with Dirichlet boundary conditions at $x=S_{T}+\eta_{2}$ on $L^{2}\left(W_{1}\right)$.

We extend $V \mid W_{2}$ in a continuous manner to potential $\widetilde{V}_{2}$ on $\mathbb{R}$ such that $\tilde{V}_{2}=\mathcal{O}(|x|)$ and $\tilde{V}_{2} \rightarrow \infty$ as $x \rightarrow-\infty$ and for some $\varepsilon_{2}>0, \varepsilon_{1} \geqq \varepsilon_{2}>0$, $\left.\left(\tilde{V}_{2}-\tilde{e}_{0}\right) \mid\left(-\infty, S_{T}+\eta_{1}\right)\right]>\varepsilon_{2}>0$. Let $H_{2}(h, F)$ be the self-adjoint operator on $L^{2}(\mathbb{R})$ with symbol $h^{2} p^{2}+\widetilde{V}_{2}$ and core $C_{0}^{\infty}(\mathbb{R}) ; \sigma\left(H_{2}\right)$ is purely descrete and each eigenvalue has multiplicity one.

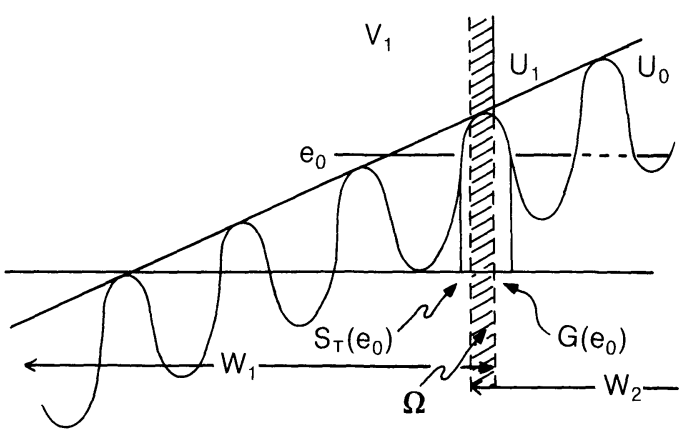

Fig. 2.1. The Stark ladder potential $V$ and regions $W_{1}, W_{2}$ and $\Omega$ 
The approximate Hamiltonian $H_{0}(h, F)$ is defined to be the direct sum $H_{1}(h, F) \oplus H_{2}(h, F)$ on the Hilbert space $\mathscr{H}_{0} \equiv L^{2}\left(W_{1}\right) \oplus L^{2}(\mathbb{R})$. It follows as in Lemma 2.1 that $\sigma\left(H_{0}\right)=\mathbb{R}$ for $F>0$ and that $H_{0}$ has infinitely many embedded simple eigenvalues. We need an estimate on the spacing of the eigenvalues of $\mathrm{H}_{2}$ below the energy $V(0)=v(0)=v_{0}$.

Lemma 2.2. Let $\tilde{e}_{0} \in \sigma\left(\tilde{h}_{0}\right)$ as described above. Then there exists an interval $I=\left[I^{-}, I^{+}\right]$such that $\tilde{e}_{0} \in I$ and if $F>0$, we have $\operatorname{dist}\left(I^{ \pm}, \sigma\left(H_{2}\right)\right)>c F h^{2+\varepsilon}$ for any $\varepsilon>0$, and $\sigma\left(\mathrm{H}_{2}\right) \cap I \neq \varnothing$.

Proof. Let $N$ be the number of unit cells between $x=0$ and $x=S_{T}\left(\tilde{e}_{0}\right)$ as given in (2.2). For each cell located in $I_{k} \equiv[-k \tau,-(k-1) \tau], k=1, \ldots, N-1$ we define a single-cell Hamiltonian $\tilde{h}_{k}$ as follows.

Let $\widetilde{V}_{k}=V \mid I_{k}$ and extend $\widetilde{V}_{k}$ in a continuous monotonically increasing way to a potential $\tilde{V}_{k}$ on $\mathbb{R}$ such that $\lim _{|x| \rightarrow \infty} \widetilde{V}_{k}(x)=\infty$ and $\widetilde{V}_{k}(x)=\mathcal{O}(|x|)$. We can arrange things such that

$$
\tilde{V}_{k}=\tilde{V}_{0}-k F \tau, \quad k=0, \ldots, N-2,
$$

where $\tilde{V}_{0}$ is defined above. For the $N^{\text {th }}$ unit cell, let $\tilde{V}_{N-1}$ be a potential on $\mathbb{R}$ such that $\widetilde{V}_{N-1}\left|\left(-\infty, S_{T}\left(\tilde{e}_{0}\right)+\eta_{2}\right]=V_{2}, \tilde{V}_{N-1}\right|\left[S_{T}\left(\tilde{e}_{0}\right)+\eta_{2},-(N-1) \tau\right]=V$, and $\tilde{V}_{N-1}$ is monotonically increasing on $[-(N-1) \tau, \infty)$. Let $\tilde{h}_{k} \equiv h^{2} p^{2}+\tilde{V}_{k}$ on $L^{2}(\mathbb{R})$. It follows from (2.3) that $\sigma\left(\tilde{h}_{k}\right)=\sigma\left(\tilde{h}_{0}\right)-k F \tau, k=0, \ldots, N-2$. Let $N_{k}\left(v_{0}\right)$ be the number of eigenvalues of $\tilde{h}_{k}$ below $v_{0}$. By the Bargman bound [RS2], we obtain:

$$
N_{k}\left(v_{0}\right) \leqq c h^{-2} \tau^{2}\left(v_{0}+k F \tau\right), \quad k=0, \ldots, N-1
$$
for some $c>0$. Letting $\tilde{H} \equiv \bigoplus_{i}^{N-1} \tilde{h}_{i}$ on $\bigoplus_{i=0}^{N-1} L^{2}(\mathbb{R})$ we obtain the number of eigen-
values of $\tilde{H}$ below $v_{0}, N\left(v_{0}\right):{ }_{i=0}$

$$
\begin{aligned}
N\left(v_{0}\right) & \leqq c h^{-2} \tau^{2}\left(N v_{0}+\frac{1}{2} N(N-1) F \tau\right) \\
& \geqq c\left(h^{2} F\right)^{-1},
\end{aligned}
$$

where we used (2.2) and the fact that $\tau$ is independent of $h$ and $F$. Now suppose there are no points $I^{ \pm}$as in the lemma but for $\tilde{H}$ rather than $H_{2}$. This implies that for energies less than $v_{0}$ the spacing of eigenvalues for $\tilde{H}$ must be less than $\frac{1}{2} c F h^{2+\varepsilon}$ for any $\varepsilon>0$. This implies a lower bound on $N\left(v_{0}\right)$ given by:

$$
c F^{-1} h^{-(2+\varepsilon)} \leqq N\left(v_{0}\right)
$$

which, for all $h$ sufficiently small, contradicts (2.5). Finally, we note that by [BCD3], the existence of an interval $I=\left[I^{-}, I^{+}\right]$about $\tilde{e}_{0}$ satisfying the properties of the lemma for $\tilde{H}$ implies the existence of an eigenvalue $e_{0}$ in $I$ for $H_{2}$. Moreover, the width of the gaps, dist $\left(\sigma\left(H_{2}\right) \cap I, \sigma\left(H_{2}\right) \backslash I\right)$, shifts at most by a small $\mathcal{O}\left(\exp \left(-c h^{-1}\right)\right)$, $c>0$, from that for $\tilde{H}$. This proves the lemma.

Note that the total multiplicity of $\sigma\left(H_{2}\right) \cap I$ is the same as $\sigma(\tilde{H}) \cap I$ [BCD3], which is finite. Let $Y \subset I$ be the smallest closed interval such that $\sigma\left(H_{2}\right) \cap I \subset Y$. For later use, we call a unit cell $I_{k}$ resonant at energy $e_{0}$ if $\sigma\left(\tilde{h}_{k}\right) \cap Y \neq \varnothing$. 


\section{Non-Trapping Property of the Potential}

We assume that $v$ satisfies conditions (v1) and we consider a non-threshold energy $e$, i.e. one for which $V^{\prime}\left(S_{T}(e)\right)>0$. By continuity, there exists a small interval $I_{0}=\left[I_{0}^{-}, I_{0}^{+}\right] \ni e$ such that $V^{\prime}\left(S_{T}(E)\right) \geqq \eta>0$ for all $E \in I_{0}$ and some $\eta>0$. We prove that the potential $V=v+F x$ with $F>0$ is non-trapping for all energies in a possibly smaller interval $I_{0}$ about $e$ on $\left(-\infty, S_{T}(e)+\delta_{0}\right]$, where $\delta_{0}>0$ is determined below. We use the formulation of quantum non-trapping due to [BCD4] (see also [DeBH, N and Si1]).

Definition 3.1 $A$ potential $V \in C^{1}(\mathbb{R})$ is non-trapping at the energy $E$ and on the region $\Omega \subset \mathbb{R}$ if there exists $\varepsilon_{0}>0$ and a vector field $f \in C^{1}(\mathbb{R})$ such that for all $x \in \Omega$,

$$
S_{E}(f, x) \equiv 2 f^{\prime}(x)(E-V(x))-f(x) V^{\prime}(x) \geqq \varepsilon_{0}>0 .
$$

Theorem 3.2. Assume that $v$ satisfies (v1) and that $F>0$. Let $e$ be such that $V^{\prime}\left(S_{T}(e)\right)>0$. Then there exists an interval $I_{0} \ni e$ such that for any $E \in I_{0}$, there exists a bounded vector field $f \in C^{\infty}(\mathbb{R})$, depending on $E$, with $f^{\prime}$ bounded, $f: \mathbb{R} \rightarrow \overline{\mathbb{R}^{-}}$, and constants $\delta_{0}, \varepsilon_{0}>0$ such that for any $E \in I_{0}$ and $x \in\left(-\infty, S_{T}(e)+\delta_{0}\right]$ :

$$
S_{E}(f, x) \geqq \varepsilon_{0}>0 .
$$

Moreover, supp $f \subset\left(-\infty, S_{T}(e)+\delta_{1}\right], \delta_{1}<\eta_{1}$ (defined in Sect. 2).

Proof. We define two vectors fields $f_{1}$ and $f_{2}$. Let $I_{0}=\left[I_{0}^{-}, I_{0}^{+}\right] \ni e$ be as above. Let $x_{0}<S_{T}\left(I_{0}^{-}\right), x_{0}$ will be fixed below, and for $E \in I_{0}$ define for $x<x_{0}$ :

$$
f_{1}(x) \equiv \frac{1}{2}[E-V(x)]^{-1 / 2} \int_{S_{T}\left(I_{0}^{-}\right)}^{x} a(s)[E-V(s)]^{-1 / 2} d s
$$

for any real bounded function $a(s)>0$. We also define

$$
f_{2}(x)=x
$$

(recall that $x=0$ is fixed in Sect. 2). Note that $f_{1}$ and $f_{2}$ are non-positive for $x<0$. The vector field $f$ has the form $f=\sum_{i=1}^{2} g_{i} f_{i}$ for a suitable pair of functions $0 \leqq g_{i} \leqq 1$, $i=1,2$, satisfying $g_{1}+g_{2}=1$ on $\left(-\infty, S_{T}\left(I_{0}^{+}\right)+\delta_{0}\right]$, where $\delta_{0}$ is defined below. Upon computing the virials (3.1) for $f_{i}$, we obtain (dropping the subscript $E$ which is fixed):

$$
\begin{gathered}
S\left(f_{1}, x\right)=a(x), \\
S\left(f_{2}, x\right)=-2(V(x)-E)-x V^{\prime}(x),
\end{gathered}
$$

and

$$
S(f, x)=\sum_{i=1}^{2} g_{i}(x) S\left(f_{i}, x\right)+\mathscr{E}(x),
$$

where $\mathscr{E}(x)$, the error term, is given by

$$
\mathscr{E}(x)=2(E-V(x)) g_{2}^{\prime}(x)\left(f_{2}(x)-f_{1}(x)\right) .
$$

By the assumption on $I_{0}$, we can find a possibly smaller interval $I_{0}=\left[I_{0}^{-}, I_{0}^{+}\right] \ni e($ by 


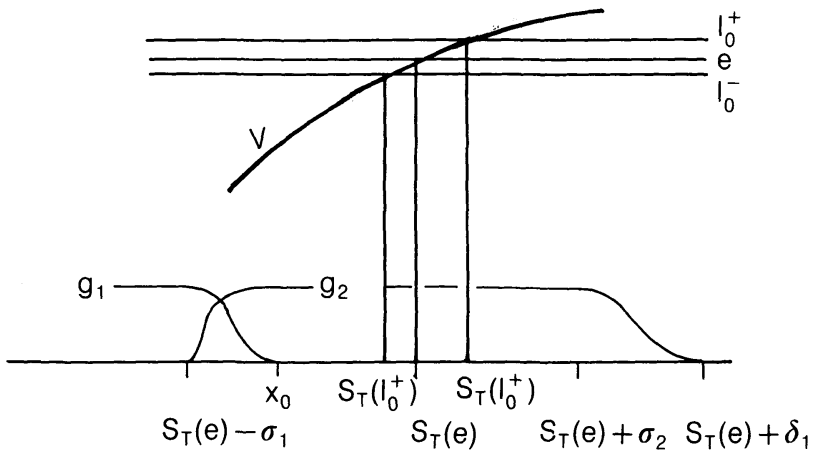

Fig. 3.1. Geometry for construction of the vector field $f$

taking $\left|I_{0}\right|$ sufficiently small) and $\sigma_{1}, \sigma_{2}>0$ such that for some $\varepsilon_{1}>0$ and $E \in I_{0}$,

$$
\begin{aligned}
S\left(f_{2}, x\right) \mid\left[S_{T}\left(I_{0}^{-}\right), S_{T}\left(I_{0}^{+}\right)\right] & \geqq \varepsilon_{1}, \\
S\left(f_{2}, x\right) \mid\left[S_{T}(e)-\sigma_{1}, S_{T}(e)+\sigma_{2}\right] & \geqq \varepsilon_{1} / 2, \\
V^{\prime}(x) \mid\left[S_{T}(e)-\sigma_{1}, S_{T}(e)+\sigma_{2}\right] & \geqq \eta / 2
\end{aligned}
$$

and $\sigma_{2}<\eta_{1}$ (defined in Sect. 2). We choose $x_{0}$ such that $S_{T}(e)-\sigma_{1}<x_{0}<S_{T}\left(I_{0}^{-}\right)$ and $g_{2} \in C^{\infty}(\mathbb{R}), 0 \leqq g_{2} \leqq 1$ such that $\operatorname{supp} g_{2}^{\prime} \subset\left[S_{T}(e)-\sigma_{1}, x_{0}\right] \cup\left[S_{T}(e)+\sigma_{2}, S_{T}(e)+\delta_{1}\right]$, where $\sigma_{2}<\delta_{1}<\eta_{1}$, and $g_{2} \mid\left[x_{0}, S_{T}(e)+\sigma_{2}\right]=1$. Then $g_{1}$ is fixed by the condition that $g_{1}+g_{2}=1$ on $\left(-\infty, S_{T}(e)+\sigma_{2}\right]$ (see Fig. 3.1). Consequently, for any $a(x) \geqq 0$, the summation term in (3.7) satisfies the bound:

$$
\left.\sum_{i=1}^{2} g_{i}(x) S\left(f_{i}, x\right)\right|_{\left(-\infty, S_{T}(e)+\sigma_{2}\right]} \geqq\left.\min \left(a(x) g_{1}(x)+\left(\varepsilon_{1} / 2\right) g_{2}(x)\right)\right|_{\left(-\infty, S_{T}(e)+\sigma_{2}\right]} .
$$

It remains to show that we can choose $a(x)$ such that (3.9) is strictly positive and such that $\mathscr{E}(x)>0$ on $\left(-\infty, S_{T}(e)+\sigma_{2}\right]$. By construction of $g_{2}, \operatorname{supp} g_{2}^{\prime} \cap$ $\left(-\infty, S_{T}(e)+\sigma_{2}\right]=\left[S_{T}(e)-\sigma_{1}, x_{0}\right]$. In this region $E-V(x)>0, E \in I_{0}, g_{2}^{\prime}(x) \geqq 0$, so it suffices to choose $a(x)$ such that $f_{2}(x)-f_{1}(x) \geqq 0$ on this region where

$$
\left(f_{2}(x)-f_{1}(x)\right)\left|\left[S_{T}(e)-\sigma_{1}, x_{0}\right] \geqq\left(S_{T}(e)-\sigma_{1}\right)+\right| f_{1}(x) \mid .
$$

We choose $a(x)$ such that

$$
a(x)=\alpha V^{\prime}(x) \geqq 0, \quad \alpha>0
$$

for $x \in\left[S_{T}(e)-\sigma_{1}, S_{T}\left(I_{0}^{-}\right)\right]$and such that

$$
a(x) \rightarrow a_{0}>0
$$

as $x \rightarrow-\infty$. It then follows from (3.3) and (3.11) that

$$
\left|f_{1}(x)\right|=\alpha\left(1-\left(\frac{E-I_{0}^{-}}{E-V(x)}\right)^{1 / 2}\right) \geqq \alpha \kappa>0
$$

on $\left[S_{T}(e)-\sigma_{1}, x_{0}\right]$ where $\kappa>0$. Hence we choose $\alpha=\kappa^{-1}\left|S_{T}(e)-\sigma_{1}\right|$ so by (3.8), 
(3.10), and (3.13), $\mathscr{E}(x)>0$. Moreover, on $\left[S_{T}(e)-\sigma_{1}, x_{0}\right], \min \left(a(x) g_{1}(x)+(\varepsilon / 2) g_{2}(x)\right)$ is strictly positive. Thus, the lower bound on $S(f, x)$ follows from (3.9), (3.11)-(3.12). Finally, we take $\delta_{0}=\sigma_{2}$.

\section{Spectral Deformation}

We construct analytic type $A$ families of operators associated with $H(h, F)$ and $H_{1}(h, F)$ (and, consequently, $\left.H_{0}(h, F)\right)$ using the vector field constructed in Sect. 3. The method of spectral decomposition using the flows generated by smooth vector fields was developed in [ $\mathrm{HiSi}$ and $\mathrm{Si} 2]$. Here, we use an infinitesimal version of the theory due to Hunziker [Hz].

Let $f: \mathbb{R} \rightarrow \widetilde{\mathbb{R}^{-}}$be the vector field constructed in Theorem 3.2. We recall that $f$ depends on $E$. For $t \in \mathbb{R}$, consider the map $\alpha_{t}: \mathbb{R} \rightarrow \mathbb{R}$ defined by

$$
\alpha_{t}(x)=x+t f(x) \text {. }
$$

Because supp $f \subset\left(-\infty, S_{T}\left(e_{0}\right)+\delta_{1}\right], \alpha_{t}$ leaves $W_{1}$ and $W_{2}$ separately invariant. Note that $\alpha_{t} \mid W_{2}$ is the identity map. The map $\alpha_{t}$ is a $C^{1}$-diffeomorphism of $\mathbb{R}$ for $|t|<M_{1}$, where $M_{1}=\left(\max _{x \in R}\left|f^{\prime}(x)\right|\right)^{-1}$. We define an operator $U_{t}, t \in \mathbb{R}$ and $|t|<M_{1}$, on $L^{2}(\mathbb{R})$ or $L^{2}\left(W_{1}\right)$ as follows (we use the same symbol to denote either operator). Let $J_{t}(x)$ be the Jacobian of $\alpha_{t}$ :

$$
J_{t}(x)=1+t f^{\prime}(x)
$$

For $g \in C_{0}^{\infty}(\mathbb{R})$ or $C_{0}^{\infty}\left(W_{1}\right)$, let

$$
\left(U_{t} g\right)(x)=J_{t}(x)^{1 / 2} g\left(\alpha_{t}(x)\right) .
$$

Since $J_{t}(x) \neq 0$ for $|t|<M_{1}$, it is easy to show that $U_{t}$ extends to a unitary transformation on $L^{2}(\mathbb{R})$ or $L^{2}\left(W_{1}\right)$ for $|t|<M_{1}$. Similarly, we define $\tilde{U}_{t}$ on $\mathscr{H}_{0} \equiv L^{2}\left(W_{1}\right) \oplus L^{2}(\mathbb{R})$ by

$$
\tilde{U}_{t}(u \oplus v)=U_{t} u \oplus v .
$$

Then $\tilde{U}_{t},|t|<M_{1}$ extends to a unitary operator on $\mathscr{H}_{0}$; again, we write $U_{t}$ for $\tilde{U}_{t}$ for simplicity.

Since $f \in C_{b}^{\infty}(\mathbb{R}), U_{t},|t|<M_{1}$, preserves the core $C_{0}^{\infty}(\mathbb{R})$ of $H(h, F)$ and the core

$$
C_{1} \equiv H^{2}\left(W_{1}\right) \cap\left\{f \in L^{2}\left(W_{1}\right) \mid f\left(S_{T}\left(e_{0}\right)+\eta_{2}\right)=0\right\} \cap D(x)
$$

of $H_{1}(h, F)$ in $L^{2}\left(W_{1}\right)$. By a simple calculation, if $p_{t} \equiv U_{t} p U_{t}^{-1}=J_{t}^{-1 / 2} p J_{t}^{-1 / 2}$, we obtain:

$$
\begin{aligned}
p_{t}^{2} & \equiv U_{t} p^{2} U_{t}^{-1}=J_{t}^{-1 / 2} p J_{t}^{-1} p J_{t}^{-1 / 2} \\
& =p J_{t}^{-2} p+G_{t}
\end{aligned}
$$

where $G_{t} \in C_{b}^{\infty}(\mathbb{R})$ for $|t|<M_{1}$ is given by:

$$
G_{t}=\frac{1}{2}\left(J_{t}^{-3} J_{t}^{\prime}\right)^{\prime}+\frac{1}{4}\left(J_{t}^{\prime} J_{t}^{-2}\right)^{2}
$$

We define distorted Hamiltonians for $t \in \mathbb{R},|t|<M_{1}$ on domains $C_{0}^{\infty}(\mathbb{R})$ and $C_{1}$, 
respectively, by

$$
\begin{aligned}
H_{t}(h, F) & \equiv U_{t} H(h, F) U_{t}^{-1} \\
& =h^{2} p_{t}^{2}+F \alpha_{t}(x)+v \circ \alpha_{t}
\end{aligned}
$$

and

$$
\begin{aligned}
H_{1, t}(h, F) & \equiv U_{t} H_{1}(h, F) U_{t}^{-1} \\
& =h^{2} p_{t}^{2}+F \alpha_{t}(x)+v \circ \alpha_{t} .
\end{aligned}
$$

We note that $J_{t}$ and $G_{t}$ extend to analytic functions on $|t|<M_{1}$ (using the principal branch of the square root).

Proposition 4.1. Let $v$ satisfy $(\mathrm{v} 1)$ and let $H_{t}(h, F)$ and $H_{1, t}(h, F), t \in \mathbb{R},|t|<M_{1}$, be as defined in (4.6) and (4.7) with domains $C_{0}^{\infty}(\mathbb{R})$ and $C_{1}$, respectively. Then $H_{t}(h, F)$ (respectively, $H_{1, t}(h, F)$ ) extends to an analytic type $A$ family of operators with domain $D(H(h, F))$ (respectively, $D\left(H_{1}(h, F)\right)$ ) on the disc $D_{\kappa} \equiv\{z \in \mathbb{C}|| z \mid<\kappa\}$, where $\kappa<\min \left(\eta, M_{1}, 1\right)$ (independent of $h$ and $\left.F\right)$ and sufficiently small ( $\eta$ is defined in $\left.(v 1)\right)$.

Proof. We give the proof for $H(h, F)$; the proof for $H_{1}(h, F)$ follows along the same lines working on the appropriate domain. By (v1), it suffices to prove that $A_{t} \equiv p_{t}^{2}+F \alpha_{t}(x)$ is an analytic family on $D(A)$, where $A \equiv p^{2}+F x$. We take $|t|<\min \left(\eta, M_{1}, 1\right)$. From the construction of $f$ in the proof of Theorem 3.2, one easily verifies that

$$
1-J_{t}^{-2}(x)=\mathcal{O}\left(t|x|^{-3 / 2}\right)
$$

as $x \rightarrow-\infty$. For any $u \in D(A)$, it follows from (4.4) that

$$
\left\|A_{t} u\right\| \leqq\|A u\|+\left\|p\left(J_{t}^{-2}-1\right) p u\right\|+c\|u\|,
$$

where $c$ is uniformly bounded in $|t|$. The second term on the right in (4.9) can be written as:

$$
\begin{aligned}
p\left(J_{t}^{-2}-1\right) p u= & \left(J_{t}^{-2}-1\right) A u-F x\left(J_{t}^{-2}-1\right) u \\
& +\left[p, J_{t}^{-2}\right] p\left(p^{2}+1\right)^{-1} A u-F\left[p, J_{t}^{-2}\right] p\left(p^{2}-1\right)^{-1} x u
\end{aligned}
$$

Using (4.10) and (4.8) in (4.9), we conclude that $D\left(A_{t}\right) \supset D(A)$. Conversely, let $w \in D\left(A_{t}\right)$. Mimicking (4.9), we have

$$
\|A w\| \leqq\left\|A_{t} w\right\|+\left\|p\left(1-J_{t}^{-2}\right) p w\right\|+c\|w\| .
$$

The second term on the right in (4.11) can be written as:

$$
\begin{aligned}
p\left(1-J_{t}^{-2}\right) p w= & \left(1-J_{t}^{-2}\right) A w+F\left(1-J_{t}^{-2}\right) x w \\
& -2 i t J_{t}^{-3} f^{\prime \prime} p\left(p^{2}+1\right)^{-1}[A w-(F x-1) w],
\end{aligned}
$$

so by (4.8) and simple bounds on $J_{t}^{-1}$ one has

$$
\left\|p\left(1-J_{t}^{-2}\right) p w\right\| \leqq c_{1}|t|\|A w\|+c_{2}\|w\| .
$$

Upon taking $|t|$ sufficiently small, (4.11)-(4.12) imply that $D(A) \supset D\left(A_{t}\right)$. Since for any $u \in D(A), t \rightarrow A_{t} u$ is analytic for $|t|$ sufficiently small, $A_{t}$ is an analytic family of type $A$. 
The family $U_{t},|t| \leqq M_{1}$, does not form a group. However, Hunziker [Hz] has shown how one can recover the standard results of the Aguilar-Balslev-Combes theory ([AC, BC]) in this setting. In particular, there exists a dense set $\mathscr{A}$ of analytic vectors for $U_{t},|t|$ sufficiently small, and for $\psi, \phi \in \mathscr{A}$, the matrix elements

$$
\left(\psi,(H(h, F)-z)^{-1} \phi\right),
$$

analytic for $\operatorname{Im} z>0$, have a meromorphic continuation into the lower-half complex plane. The poles of this continuation, which are independent of $\phi$ and $\psi$, are defined to be the spectral resonances of $H(h, F)$. These are equal to the complex eigenvalues of $H_{t}(h, F), \operatorname{Im} t>0$, which are located in the lower-half complex plane if they exist.

\section{Existence of Resonance Free Domains}

We combine the results of Sects. 3 and 4 to show that there exists a full complex neighborhood of the energy interval $I_{0} \ni e_{0}$, where $e_{0} \in \sigma\left(H_{2}\right)$ is as in Lemma 2.2 and $I_{0}$ is the corresponding interval of Theorem 3.2, which lies in the resolvent set of $H_{1, t}(h, F)$ for $\operatorname{Im} t>0$ and sufficiently small. We follow the ideas of [BCD4] (see also [DeBH, Si, N]). Until this section, our results are valid for any value of $h$. In this section, we will have to assume that $h$ is small due to the fact that the analysis presented here is restricted to the semi-classical regime.

Theorem 5.1. Assume that $v$ satisfies (v1) and that $e_{0} \in \sigma\left(H_{2}\right)$ is a NTH energy. Let $H_{1, t}(h, F), F>0,|t|$ sufficiently small, be the type $A$ analytic family of operators constructed in Sect. 4 using the vector field for energy $e_{0}$. For $t=i \beta, \beta>0$, let $\mathcal{O}_{\beta} \subset \mathbb{C}$ be defined by (see Fig. 5.1):

$$
\mathcal{O}_{\beta} \equiv\left\{z \mid\left(\varepsilon_{0} \beta / 2-\operatorname{Im} J_{i \beta}^{2}\left(e_{0}-z\right)\right)>0 \text { for all } x \in\left(-\infty, S_{T}\left(e_{0}\right)+\delta_{0}\right]\right\}
$$

where $I_{0}, \varepsilon_{0}$, and $\delta_{0}$ are defined in Theorem 3.2. Then for any $z \in \mathcal{O}_{\beta}$, one has for $F>0$ and $h$ small enough,

(1) $z \in \rho\left(H_{1, i \beta}(h, F)\right)$;

(2) $\left\|\left(H_{1, i \beta}-z\right)^{-1}\right\| \leqq c_{1}\left(\varepsilon_{0} \beta / 2-c_{2} h\right)^{-1}$ for some $c_{1}, c_{2}>0$;

(3) for any $\chi \in C_{0}^{1}\left(\left[S_{T}\left(e_{0}\right)+\eta_{1}, S_{T}\left(e_{0}\right)+\eta_{2}\right]\right)$,

$$
\left\|\chi p\left(H_{1, i t}-z\right)^{-1}\right\| \leqq c_{1} h^{-1}\left(\varepsilon_{0} \beta / 2-c_{2} h\right)^{-1} .
$$

Proof. We write $t=i \beta, \beta>0$ and small; $H_{1, i \beta}(h, F)$ will be written as $H_{1}(i \beta)$; and

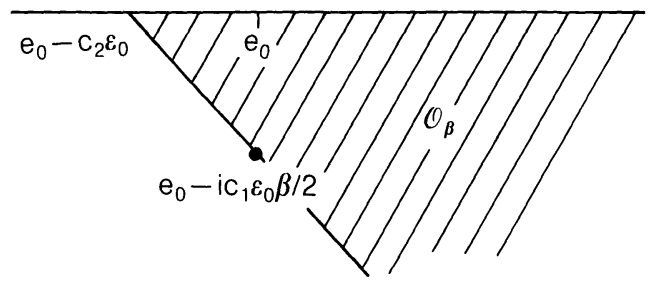

Fig. 5.1. Resonance free domain $\mathcal{O}_{\beta}$ in $\mathbb{C}$ 
we recall that $J_{i \beta}=1+i \beta f^{\prime}$. It follows by the Cauchy-Schwarz inequality that for all $u \in D\left(H_{1}(h, F)\right), F>0$,

$$
\left\|\left(H_{1}(i \beta)-z\right) J_{i \beta} u\right\| \geqq \operatorname{Im}\left\langle J_{i \beta}^{*} w,\left(H_{1}(i \beta)-z\right) J_{i \beta} u\right\rangle\left\|J_{i \beta}^{*} w\right\|^{-1}
$$

for any $w \in L^{2}\left(W_{1}\right)$. Let $\left\{\chi_{i}\right\}_{i=1}^{2}$ be a partition of unity covering $W_{1}=\left(-\infty, S_{T}\left(e_{0}\right)+\eta_{2}\right]$ with $\sum \chi_{i}^{2}=1$ on $W_{1}$ and $\chi_{1}\left|\left(-\infty, S_{T}\left(I_{0}^{+}\right)+\varepsilon_{1}\right]=1, \chi_{2}\right|\left[S_{T}\left(e_{0}\right)+\delta_{0}, S_{T}\left(e_{0}\right)+\eta_{2}\right]=1$ so supp $\chi_{i}^{\prime} \subset\left[S_{T}\left(I_{0}^{+}\right)+\varepsilon_{1}, S_{T}\left(e_{0}\right)+\delta_{0}\right]$ for any $0<\varepsilon_{1}<\delta_{0}$. We take $w=-\left(\chi_{1}^{2}+i \chi_{2}^{2}\right) u$ and consider the inner product in (5.1). It can be expanded as

$$
\begin{gathered}
-\operatorname{Im}\left\langle u, \chi_{1} J_{i \beta}\left(H_{1}(i \beta)-z\right) J_{i \beta} \chi_{1} u\right\rangle \\
-\operatorname{Im} h^{2}\left\langle u, \chi_{1}\left[\chi_{1}, p^{2}\right] u\right\rangle \\
+\operatorname{Re}\left\langle u, \chi_{2} J_{i \beta}\left(H_{1}(i \beta)-z\right) J_{i \beta} \chi_{2} u\right\rangle \\
+\operatorname{Re} h^{2}\left\langle u, \chi_{2}\left[\chi_{2}, p^{2}\right] u\right\rangle,
\end{gathered}
$$

where, in evaluating the terms (5.3) and (5.5), we use the fact that $f \mid \operatorname{supp} \chi_{i}^{\prime}=f_{2}$. We first consider the error terms (5.3) and (5.5). By expanding the commutators in (5.3) and (5.5) and using the basic fact that

$$
\operatorname{Re}\left\langle u,\left(f D_{x}+D_{x} f\right) u\right\rangle=0
$$

for $f \in C^{1}$ and $u \in D\left(D_{x}\right), D_{x}=\frac{d}{d x}$, we obtain

$$
\left|\operatorname{Re} h^{2}\left\langle u, \chi_{2}\left[\chi_{2}, p^{2}\right] u\right\rangle\right| \leqq \operatorname{ch}^{2}\|u\|^{2}
$$

and

$$
\left|\operatorname{Im} h^{2}\left\langle u, \chi_{1}\left[\chi_{1}, p^{2}\right] u\right\rangle\right| \leqq c_{1} h^{2}\|u\|^{2}+c_{2} h^{2}\left\|\chi_{1}^{\prime} p u\right\|\|u\| .
$$

As for the last term in (5.7), note that supp $\chi_{1}^{\prime}$ is compact and that $f \mid \operatorname{supp} \chi_{1}^{\prime}=x$. Consequently, we have

$$
h \chi_{1}^{\prime} p=i h \chi_{1}^{\prime \prime}+h p\left(h^{2} p^{2}+1\right)^{-1}\left\{\chi_{1}^{\prime}\left(h^{2} p^{2}+1\right)+h^{2}\left[p^{2}, \chi_{1}^{\prime}\right]\right\}
$$

and

$$
\chi_{1}^{\prime}\left(h^{2} p^{2}+1\right)=(1+i \beta)^{2} \chi_{1}^{\prime}\left\{\left(H_{1}(i \beta)-z\right)+(z+r(x, \beta))\right\}
$$

where $\chi_{1}^{\prime} r$ is uniformly bounded for small $\beta$. Since $(h p)^{n}\left(h^{2} p^{2}+1\right)^{-1}$ is uniformly bounded for $n=0,1,2$, it follows from $(5.8 \mathrm{a}, \mathrm{b})$ that

$$
\left|\operatorname{Im} h^{2}\left\langle u, \chi_{1}\left[\chi_{1}, p^{2}\right] u\right\rangle\right| \leqq c_{1} h\|u\|^{2}+c_{2} h\left\|\left(H_{1}(i \beta)-z\right) J_{i \beta} u\right\|\|u\| .
$$

We next treat (5.2) by first separating kinetic and potential energy terms. It follows from (4.4) that $J_{i \beta} p_{i \beta}^{2} J_{i \beta}=p^{2}+\tilde{G}_{i \beta}$ so the kinetic energy contribution to (5.2) is

$$
h^{2} \operatorname{Im} \chi_{1} J_{i \beta} p_{i \beta}^{2} J_{i \beta} \chi_{1}=h^{2} \operatorname{Im} \chi_{1}^{2} \widetilde{G}_{i \beta} .
$$

As for the potential energy, a simple calculation shows that

$$
-\operatorname{Im} J_{i \beta}^{2}\left(V_{i \beta}-z\right) \chi_{1}^{2}=\left[\beta S_{e_{0}}(f, x)-\operatorname{Im} J_{i \beta}^{2}\left(e_{0}-z\right)\right] \chi_{1}^{2}+\mathcal{O}\left(F^{2} \beta^{3}\right) \chi_{1}^{2},
$$

where we used the fact that $f$ and $V^{\prime \prime}$ are bounded on supp $\chi_{1}$. By Theorem 3.2, 
$S_{E}(f, x) \chi_{1}^{2} \geqq \varepsilon_{0} \chi_{1}^{2}$ for $E \in I_{0}$, so by the definition of $\mathcal{O}_{\beta}$,

$$
-\operatorname{Im} J_{i \beta}^{2}\left(V_{i \beta}-z\right) \chi_{1}^{2} \geqq\left(\varepsilon_{0} \beta / 2\right) \chi_{1}^{2}-\mathcal{O}\left(\beta^{3} F^{2}\right) \chi_{1}^{2} .
$$

Combining this with (5.10), we obtain

$$
-\operatorname{Im} \chi_{1} J_{i \beta}\left(H_{1}(i \beta)-z\right) J_{i \beta} \chi_{1} \geqq\left[\varepsilon_{0} \beta / 2-h^{2} \operatorname{Im} \tilde{G}_{i \beta}-\mathcal{O}\left(F^{2} \beta^{3}\right)\right] \chi_{1}^{2} .
$$

Finally, we treat (5.4). As above, the kinetic energy contribution is

$$
\begin{aligned}
\operatorname{Re}\left\langle u, \chi_{2} J_{i \beta}\left(h^{2} p_{i \beta}^{2}\right) J_{i \beta} \chi_{2} u\right\rangle & =h^{2}\left\langle u, \chi_{2} p^{2} \chi_{2} u\right\rangle+h^{2} \operatorname{Re}\left\langle u, \chi_{2}^{2} \tilde{G}_{i \beta} u\right\rangle \\
& \geqq h^{2} \operatorname{Re}\left\langle u, \chi_{2}^{2} \widetilde{G}_{i \beta} u\right\rangle .
\end{aligned}
$$

The potential energy term for $x \in W_{1}$ yields

$$
\begin{aligned}
\operatorname{Re}\left(V_{i \beta}-z\right) J_{i \beta}^{2} \chi_{2}^{2} & =(V-\operatorname{Re} z)\left(1-\beta^{2}\left(f^{\prime}\right)^{2}\right) \chi_{2}^{2}-2 \beta f^{\prime}\left(f V^{\prime} \beta-\operatorname{Im} z\right) \chi_{2}^{2}-\mathcal{O}\left(\beta^{2}\right) \chi_{2}^{2} \\
& \geqq \frac{1}{2}(V-\operatorname{Re} z) \chi_{2}^{2}-\beta^{2}\left(c_{2}+F c_{3}\right) \chi_{2}^{2},
\end{aligned}
$$

since $\operatorname{Im} z>-c \beta \varepsilon_{0}$ for $z \in \mathcal{O}_{\beta}$. We choose $\varepsilon_{1}$ as above, i.e. $0<\varepsilon_{1}<\delta_{0}$, which implies that for $x \in W_{1}$,

$$
(V-\operatorname{Re} z) \chi_{2}^{2} \geqq c \varepsilon_{1} \chi_{2}^{2},
$$

for $z \in \mathcal{O}_{\beta}$ (we used the NTH condition to estimate $\left.\left(V-I_{0}^{+}\right) \chi_{2}^{2} \geqq c \varepsilon_{1} \chi_{2}^{2}\right)$. It follows from $(5.15-5.16)$ that

$$
\operatorname{Re}\left(V_{i \beta}-z\right) J_{i \beta}^{2} \chi_{2}^{2} \geqq c \chi_{2}^{2} \geqq 0
$$

for $\beta$ sufficiently small. Using (5.13), (5.14) and (5.17), we obtain a lower bound for (5.2) and (5.4),

$$
\left(\varepsilon_{0} \beta / 2-c_{2} F^{2} \beta^{2}\right)\left\|\chi_{1} u\right\|^{2}-c_{2} h^{2}\|u\|^{2}+c_{3}\left\|\chi_{2} u\right\|^{2} \geqq\left(\varepsilon_{0} \beta / 2-c h^{2}\right)\|u\|^{2},
$$

provided $\beta$ and $h$ are small. The error terms (5.3) and (5.5) are estimated in (5.6) and (5.9) and contribute

$$
-c_{1} h\|u\|^{2}-c_{2} h\|u\|\left\|\left(H_{1}(i \beta)-z\right) J_{i \beta} u\right\| .
$$

Also note that $\left\|J_{i \beta}^{*} w\right\|<c\|u\|$, since $f^{\prime}$ is bounded. Combining (5.18-5.19) on the right side of (5.1) and using the facts that $J_{i \beta}$ is an invertible map on $L^{2}\left(W_{1}\right)$ with $\left\|J_{i \beta}^{-1} u\right\| \geqq c\|u\|$, we obtain

$$
\left\|\left(H_{1}(i \beta)-z\right) u\right\| \geqq c_{1}\left(\varepsilon_{0} \beta / 2-c_{2} h\right)\|u\| .
$$

As it is easy to check that a similar estimate holds for $H_{1}(i \beta)^{*}-\bar{z}, z \in \mathcal{O}_{\beta}$, it follows that $\mathcal{O}_{\beta} \subset \rho\left(H_{1}(i \beta)\right)$ for $\beta$ sufficiently small and that the bound in part (2) holds. As for part (3), let $\chi$ be as stated there. Since $f \mid \operatorname{supp} \chi=0$, we have

$$
h^{2} \chi p^{2} \chi=\chi\left(H_{1}(i \beta)-z\right) \chi+\chi^{2} k(x ; \beta)
$$

$k \in C_{0}^{\infty}(\mathbb{R})$. For any $u \in L^{2}\left(W_{1}\right)$ and $\mathbf{z} \in \mathcal{O}_{\beta}$ :

$$
\begin{aligned}
\left\langle R(z) u, \chi h^{2} p^{2} \chi R(z) u\right\rangle & =\|h p \chi R(z) u\|^{2} \\
& \leqq\left\langle\chi R(z) u,\left(H_{1}(i \beta)-z\right) \chi R(z) u\right\rangle+c\|R(z)\|^{2}\|u\|^{2},
\end{aligned}
$$

where $c$ is uniformly bounded in $\beta$. The first term on the right of (5.21) becomes

$$
\langle\chi R(z) u, \chi u\rangle-2 i h\left\langle\chi^{\prime} R(z) u, \chi h p R(z) u\right\rangle+h^{2}\left\langle\chi R(z) u, \chi^{\prime \prime} R(z) u\right\rangle
$$


which is bounded above by

$$
c_{1} h\|\chi h p R(z) u\|^{2}+c_{2}\|u\|^{2}+c_{3}\|R(z)\|^{2}\|u\|^{2}
$$

for $h$ sufficiently small. Combining (5.21) with (5.22) the result follows.

By the definition of $\mathcal{O}_{\beta}$, dist $\left(\partial \mathcal{O}_{\beta}, e_{0}\right)=\mathcal{O}(F)$ and, consequently, we can choose $I^{ \pm}$as in Lemma 2.2 , such that $I \equiv\left[I^{-}, I^{+}\right] \subset \mathcal{O}_{\beta}$, $\operatorname{dist}\left(Y, I^{ \pm}\right) \geqq c h^{2+\varepsilon}$, where $Y$ is the smallest closed interval containing $\sigma\left(\mathrm{H}_{2}\right)$ near $e_{0}$.

\section{Existence of Resonances}

We prove that $H_{t}(h, F)$ has an eigenvalue $z_{0}$ in the lower-half plane for $\operatorname{Im} t>0$ with $\left|z_{0}-e_{0}\right| \leqq c e^{-\kappa / h}$ for some $0<\kappa$ and $h$ sufficiently small. Because of the periodicity of $v$, this establishes the existence of a ladder of resonances associated with $e_{0}$. We begin with a brief discussion of a geometric perturbation method developed in [BCD3] and then apply it to the resonance problem. We conclude this section by proving the crude bound on $\left|z_{0}-e_{0}\right|$ mentioned above. This will be improved in the next section. We recall that $e_{0}$ is the eigenvalue of $\mathrm{H}_{2}$ defined in Lemma 2.2. It is associated with the lowest eigenvalue of the single cell Hamiltonian $h_{0}$. We assume that $e_{0}$ is NTH and we choose the vector field $f$ to be the one constructed in Theorem 3.2 for $e=e_{0}$. Since $\left|\tilde{e}_{0}-e_{0}\right|$ is exponentially small in $h$ and $\tilde{e}_{0}$ is given by the harmonic approximation for $\tilde{V}_{0}$, (in the simplest case), the NTH condition for $e_{0}$ is satisfied for small $h$ provided it is satisfied for $\min _{x} \widetilde{V}_{0}(x)$. It is clear that this is NTH for almost all values of $F$. Let $\mathcal{O}_{\beta}$ be the resonance free domain of Theorem 5.1 and recall the choice of $I$ made there. By Lemma 2.2 one can find an interval $I=\left[I^{-}, I^{+}\right] \subset \mathcal{O}_{\beta}$ such that $e_{0} \in I$ and $\operatorname{dist}\left(I^{ \pm}, \sigma\left(H_{2}\right)\right)>c h^{2+\varepsilon}, \varepsilon, c>0$. Furthermore we may choose $I \subset I_{0}$ where $I_{0}$ is as in Theorem 3.2.

Recall from Sect. 2 that $\mathscr{H}_{0} \equiv L^{2}\left(W_{1}\right) \oplus L^{2}(\mathbb{R})$ and that $H_{0}(h, F)=H_{1}(h, F) \oplus$ $H_{2}(h, F)$. After spectral deformation, $H_{0, t}=H_{1, t} \oplus H_{2}$ is an analytic family for $|t|$ sufficiently small. Let $\left\{J_{i}\right\}_{i=1}^{2}$ be an almost everywhere differentiable partition of unity subordinate to the cover of $\mathbb{R}$ given by $W_{1}$ and $W_{2}$ such that $\sum_{i=1}^{2} J_{i}=1$.

These functions will be specified in more detail below; here we note that $\operatorname{supp} J_{i}^{\prime} \subset W_{1} \cap W_{2} \subset G\left(I^{+}\right)$, the classically forbidden region for energy $I^{+}$. Define $J: \mathscr{H}_{0} \rightarrow \mathscr{H} \equiv L^{2}(\mathbb{R})$ by

$$
J\left(u_{1} \oplus u_{2}\right)=J_{1} u_{1}+J_{2} u_{2} .
$$

Let $\left\{\tilde{J}_{i}\right\}_{i=1}^{2}$ be two almost everywhere differentiable functions such that $\tilde{J}_{i} J_{i}=J_{i}$, $i=1,2$, supp $\widetilde{J}_{1} \subset W_{1}$, and define $\widetilde{J}: \mathscr{H}_{0} \rightarrow \mathscr{H}$ in a manner analogous to (6.1). Then $\tilde{J}^{*}=\mathscr{H} \rightarrow \mathscr{H}_{0}$ satisfies

$$
J \tilde{J}^{*}=1_{\mathscr{H}} .
$$

Let $z \in \rho\left(H_{0, t}\right) \cap \rho\left(H_{t}\right)$, for some $t \in \mathbb{C}$, and let $R(z) \equiv\left(H_{t}-z\right)^{-1}, R_{0}(z) \equiv\left(H_{0, t}-z\right)^{-1}$. Then we have:

$$
\begin{aligned}
R(z) J & =J R_{0}(z)+R(z)\left(J H_{0, t}-H_{t} J\right) R_{0}(z) \\
& =J R_{0}(z)+R(z) \tilde{J} M R_{0}(z),
\end{aligned}
$$


where $M: \mathscr{H}_{0} \rightarrow \mathscr{H}_{0}$, defined as a quadratic form on $\mathscr{H}_{0}^{1}\left(W_{1}\right) \cap D\left(J_{1}^{\prime}\right) \oplus H^{1}(\mathbb{R}) \cap$ $D\left(J_{2}^{\prime}\right)$, is given by:

$$
M\left(u_{1} \oplus u_{2}\right)=h^{2}\left(D_{x} J_{1}^{\prime}-J_{1}^{\prime} D_{x}\right) u_{1} \oplus h^{2}\left(D_{x} J_{2}^{\prime}-J_{2}^{\prime} D_{x}\right) u_{2} .
$$

Here we used the fact that on $\Omega \equiv W_{1} \cap W_{2}$, the vector field $f=0$. Equation (6.3) is the geometric resolvent equation.

Because of the form of $\mathscr{H}_{0}$ we can write $R_{0}(z)=R_{1}(z) \oplus R_{2}(z)$. It is clear from (6.3) and (6.4) that we need to estimate terms of the form $J_{i}^{\prime} p R_{i}(z)$ and $J_{i}^{\prime \prime} R_{i}(z)$, $i=1,2$, (provided $J_{i} \in C^{2}$ ) for $z$ on a simple closed contour $\Gamma$ lying in $\mathcal{O}_{\beta}$ about $e_{0}$. From Theorem 5.1, we have good bounds on $R_{1}(z)$ for $z \in \mathcal{O}_{\beta}$ in the sense that $h^{2}\left\|J_{1}^{\prime} p R_{1}(z)\right\| \rightarrow 0$ as $h \rightarrow 0$. However, using Lemma 2.2 alone, similar bounds for $R_{2}(z)$ grow as $h \rightarrow 0$. (Unless, of course, we know more about $\sigma\left(H_{2}\right)$, for example, $\operatorname{dist}\left(I_{0}^{ \pm}, \sigma\left(H_{2}\right)\right)>c h$.) Consequently, we will write (6.3) in a form which allows us to easily use the fact that supp $J_{i}^{\prime} \subset G\left(I^{+}\right)$to obtain better estimates on the localized resolvent $J_{2}^{\prime} R_{2}(z)$.

Let $d_{A}^{E}(x, y)$ be the distance between $x$ and $y$ in the Agmon pseudo-metric at energy $E$ given by

i.e.,

$$
d s_{A}^{2} \equiv h^{-2}(V(x)-E)_{+} d x^{2},
$$

$$
d_{A}^{E}(x, y)=h^{-1} \int_{x}^{y}[V(s)-E]_{+}^{1 / 2} d s .
$$

For any open set $U \subset \mathbb{R}$, we define

$$
d_{A}(x, U) \equiv \inf _{y \in U} d_{A}^{I^{+}}(x, y) .
$$

In particular, for any $\Omega \subset G\left(I^{+}\right)$(we consider, as above, the connected component of $G\left(e_{0}\right)$ such that $S_{T}\left(e_{0}\right) \in \overline{G\left(e_{0}\right)}$ and then $\left.G\left(I^{+}\right) \subset G\left(e_{0}\right)\right)$ we will write:

$$
d_{\Omega}(x) \equiv d_{A}(x, \Omega) \text {. }
$$

Note that $d_{A}(x, U)$ is differentiable almost everywhere and

$$
\left|d_{A}^{\prime}(x, U)\right|^{2} \leqq h^{-2}\left(V(x)-I^{+}\right)_{+} \text {a.e. }
$$

(see, for example [HiSi] or [BCD3]).

We define two "wells" as follows: $\mathscr{V}_{1} \equiv\left(-\infty, S_{T}\left(I^{+}\right)\right]$and $\mathscr{V}_{2} \equiv\left[\tilde{S}_{T}\left(I^{+}\right), \infty\right)$, where $\tilde{S}_{T}\left(I^{+}\right) \equiv \inf \left\{x>S_{T}\left(I^{+}\right) \mid V(x)=I^{+}\right\}$, i.e. $\mathscr{V}_{2}$ starts at the first interior well

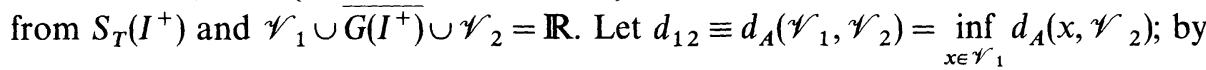
construction $d_{12} \geqq \delta_{0}>0$, some $\delta_{0}$. For the functions $J_{i}$ introduced above, we take the following. For $\delta<\frac{1}{2} d_{12}$ sufficiently small, let

$$
j(s)= \begin{cases}0 & s>\frac{1}{2} d_{12} \\ 1 & s<\frac{1}{2} d_{12}-\delta \\ \delta^{-1}\left(\frac{1}{2} d_{12}-s\right) & \frac{1}{2} d_{12}-\delta<s<\frac{1}{2} d_{12}\end{cases}
$$

and take $J_{1}(x) \equiv j\left(d_{A}\left(x, \mathscr{V}_{1}\right)\right)$ and we set $J_{2}(x)=1-J_{1}(x)$. Note that supp $J_{i}^{\prime} \subset G\left(I^{+}\right)$ and that for $i=1,2$ :

$$
\left|J_{i}^{\prime}(x)\right|^{2} \leqq h^{-2} \delta^{-2}\left(V(x)-I^{+}\right)_{+} \text {a.e. }
$$


We now take $W_{1}$ and $W_{2}$ (i.e. we fix the points $\eta_{1}$ and $\eta_{2}$ as defined in Sect. 2) such that $\Omega=W_{1} \cap W_{2} \subset\left\{x \in G\left(I^{+}\right) \mid \frac{1}{2} d_{12}-\delta<d_{A}\left(\mathscr{V}_{1}, x\right)<\frac{1}{2} d_{12}\right\} \subset G\left(I^{+}\right)$, for $\delta$ sufficiently small; $\delta$ will be adjusted below. Also note that $d_{\Omega}^{I^{+}}=\inf _{x \in G\left(I^{+}\right)^{c}} d_{A}(x, \Omega)>0$ for $\delta$ sufficiently small. We let $\chi_{\Omega}$ be the characteristic function on $\Omega$.

Returning to (6.3) and (6.4), we factorize $M$ using two operators $M_{1}$ and $M_{2}$ defined as follows. These are operators $M_{i}: D\left(M_{i}\right) \subset \mathscr{H}_{0} \rightarrow \mathscr{H}_{0} \oplus \mathscr{H}_{0}$, where the domain is

$$
D\left(M_{i}\right) \equiv\left(L^{2}\left(W_{1}\right) \cap H^{1}(\Omega) \cap D\left(J_{1}^{\prime}\right)\right) \oplus\left(L^{2}(\mathbb{R}) \cap H^{1}(\Omega) \cap D\left(J_{2}^{\prime}\right)\right),
$$

and for $u_{1} \oplus u_{2} \in D\left(M_{i}\right)$ :

$$
\begin{aligned}
& M_{1}\left(u_{1} \oplus u_{2}\right) \equiv h\left(J_{1}^{\prime} u_{1} \oplus \chi_{\Omega} u_{1}^{\prime}\right) \oplus h\left(J_{2}^{\prime} u_{2} \oplus \chi_{\Omega} u_{2}^{\prime}\right), \\
& M_{2}\left(v_{1} \oplus v_{2}\right) \equiv h\left(\chi_{\Omega} u_{1}^{\prime} \oplus J_{1}^{\prime} u_{1}\right) \oplus h\left(\chi_{\Omega} u_{2}^{\prime} \oplus J_{2}^{\prime} u_{2}\right)
\end{aligned}
$$

It is then an easy calculation to show that

$$
M=-M_{2}^{*} M_{1},
$$

as a quadratic form on $D\left(M_{i}\right)$.

Lemma 6.1. $\operatorname{Ran} R_{0}(z) \subset D\left(M_{1}\right), z \in \rho\left(H_{0, t}\right)$ and $\operatorname{Ran} J R(z) \subset D\left(M_{2}\right), z \in \rho\left(H_{t}\right)$.

Proof. The first inclusion follows from the facts that $H_{1, t}$ and $H_{2}$ are bounded relative to $p^{2}+F x$ and that $D\left(p^{2}+F x\right) \subset H^{1}$ (where we work on $W_{1}$ and $\mathbb{R}$, respectively) and since $J_{i}$ is a Lipschitz continuous function, $J_{i}^{\prime}$ has compact support and is measurable. As for the second inclusion, it follows by similar considerations.

Lemma 6.1 allows us to use the factorization (6.15) in (6.3) which yields

$$
R(z)=J R_{0}(z)-R(z) J M_{2}^{*} M_{1} R_{0}(z) .
$$

To solve this equation formally, we write

$$
R(z) J M_{2}^{*}=J R_{0}(z) M_{2}^{*}-R(z) J M_{2}^{*} M_{1} R_{0}(z) M_{2}^{*}
$$

or

$$
R(z) J M_{2}^{*}=J R_{0}(z) M_{2}^{*}(1-K(z))^{-1},
$$

where $K(z): \mathscr{H}_{0} \oplus \mathscr{H}_{0} \rightarrow \mathscr{H}_{0} \oplus \mathscr{H}_{0}$ is defined by:

$$
K(z) \equiv-M_{1} R_{0}(z) M_{2}^{*} \text {. }
$$

Note that a sufficient condition for (6.18) is that $\|K(z)\|<1$ for $z \in \Gamma$. Using (6.18) in the right side of (6.16) and multiplying on the right by $\widetilde{J}^{*}$, we obtain, by (6.2), the desired equation on $\mathscr{H}$ :

$$
R(z)=J R_{0}(z) \tilde{J}^{*}-J R_{0}(z) M_{2}^{*}(1-K(z))^{-1} M_{1} R_{0}(z) \tilde{J}^{*} .
$$

In light of Lemma 6.1, this equation is valid as a quadratic form on $\mathscr{H}$ for $z \in \rho\left(H_{0, t}\right) \cap \rho\left(H_{t}\right)$.

We next obtain estimates on $K(z)$ for $z \in \Gamma$, where $\Gamma \subset \mathbb{C}$ is a simple closed path lying in $\mathcal{O}_{\beta}$ for $\operatorname{Im} z<0$ and passing through the endpoints $I^{ \pm}$of the interval $I \ni e_{0}$ described above; see Fig. 6.1. Note that we can choose $\Gamma$ such that $|\Gamma|=.\operatorname{ch}^{2+\varepsilon}$. 


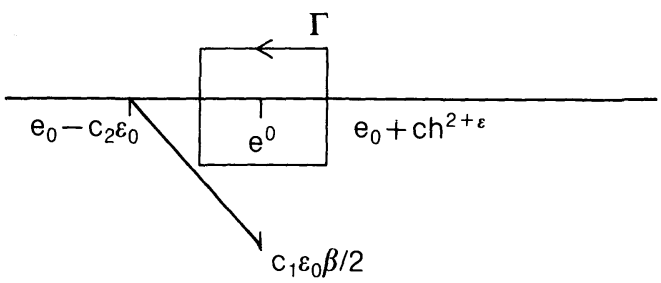

Fig. 6.1. Integration contour in $\mathcal{O}_{\beta}$

These estimates will prove that $\|K(z)\|<1$ uniformly on $\Gamma$ for $h$ sufficiently small. They will also be sufficient to prove that $\lim _{h \rightarrow 0}\left\|R(z)-J R_{0}(z) \tilde{J}^{*}\right\|=0$ uniformly on $\Gamma$.

Recall that $\Omega=W_{1} \cap W_{2} \subset G\left(I^{+}\right)$. We define a positive self-adjoint operator $C_{\Omega}$ as follows. Let $a_{\Omega}^{I^{+}}[v]$ be the quadratic form on $H^{1}(\Omega) \equiv\left\{u \in \mathscr{H} \mid u^{\prime}\right.$ exists a.e. and $\left.u^{\prime} \in L^{2}(\Omega)\right\}$ given by

$$
a_{\Omega}^{I^{+}}[v] \equiv h^{2}\left\|v^{\prime}\right\|_{\Omega}^{2}+\left\|\left(V-I^{+}\right)_{+}^{1 / 2} v\right\|_{\Omega}^{2},
$$

where $\|u\|_{\Omega}^{2} \equiv \int_{\Omega}|u(x)|^{2} d x$. By the second representation theorem [Ka], there exists a positive self-adjoint operator $C_{\Omega}$ such that $D\left(C_{\Omega}\right)=H^{1}(\Omega)$ and $\left\|C_{\Omega} v\right\|^{2}=a_{\Omega}^{I^{+}}[v]$ $\forall v \in H^{1}(\Omega)$. It follows as in [BCD3] that $D(H) \subset H^{1}(\Omega)$.

Lemma 6.2. Let $z \in \rho\left(H_{1, t}\right) \cap \rho\left(H_{2}\right)$. Then for $\delta<1$ :

$$
\|K(z)\| \leqq 2 \delta^{-1} \max _{i=1,2}\left\|C_{\Omega} R_{i}(z) C_{\Omega}\right\| .
$$

Proof. Let $u_{1} \oplus u_{2} \in D\left(M_{i}\right), i=1$ or 2 . From (6.13) and (6.14) we have for $i=1$ or 2 :

$$
\begin{aligned}
\left\|M_{i}\left(u_{1} \oplus u_{2}\right)\right\|^{2} & \leqq h^{2} \sum_{j=1}^{2}\left(\left\|u_{j}^{\prime}\right\|_{\Omega}^{2}+\left\|J_{j}^{\prime} u_{j}\right\|_{\Omega}^{2}\right) \\
& \leqq \delta^{-2} \sum_{j=1}^{2}\left(\delta^{2} h^{2}\left\|u_{j}^{\prime}\right\|_{\Omega}^{2}+\left\|\left(V-I^{+}\right)_{+}^{1 / 2} u_{j}\right\|_{\Omega}^{2}\right) \\
& \leqq \delta^{-2} \sum_{j=1}^{2}\left\|C_{\Omega} u_{j}\right\|^{2},
\end{aligned}
$$

where we used (6.11). Consequently, Lemma 6.1 and (6.23) give

$$
\left\|M_{i} R_{0}(z)\left(u_{1} \oplus u_{2}\right)\right\|^{2} \leqq \delta^{-2} \sum_{j=1}^{2}\left\|C_{\Omega} R_{j}(z) u_{j}\right\|^{2} .
$$

Next, let $\tilde{C}_{\Omega}$ denote $C_{\Omega} \oplus C_{\Omega}$ on its natural domain in $\mathscr{H}_{0}$ and note that $\left\|\tilde{C}_{\Omega} R_{0}(z) M_{i}^{*}\right\|^{2}=\left\|M_{i} R_{0}(z)^{*} \tilde{C}_{\Omega}\right\|^{2}$, so that if we let $y=y_{1} \oplus y_{2} \equiv M_{2}^{*}\left(w_{1} \oplus w_{2}\right)$, $w_{i} \in \mathscr{H}_{0} \oplus \mathscr{H}_{0}$, it follows from (6.24) that

$$
\begin{aligned}
\left\|M_{1} R_{0}(z) M_{2}^{*}\left(w_{1} \oplus w_{2}\right)\right\|^{2} & \leqq \delta^{-2} \sum_{j=1}^{2}\left\|C_{\Omega} R_{j}(z) y_{j}\right\|^{2} \\
& \leqq \delta^{-2}\left\|\tilde{C}_{\Omega} R_{0}(z) M_{2}^{*}\right\|^{2}\left\|w_{1} \oplus w_{2}\right\|^{2}
\end{aligned}
$$




$$
\begin{aligned}
& \leqq \delta^{-2}\left\|M_{2} R_{0}(z)^{*} \tilde{C}_{\Omega}\right\|^{2}\left\|w_{1} \oplus w_{2}\right\|^{2} \\
& \leqq 2 \delta^{-2}\left(\max _{i=1,2}\left\|C_{\Omega} R_{i}(z) C_{\Omega}\right\|^{2}\right)\left\|w_{1} \oplus w_{2}\right\|^{2},
\end{aligned}
$$

where we used (6.24) to evaluate the norm on the second to last line. This proves the lemma.

We use a result of [BCD3] to estimate $\left\|C_{\Omega} R_{i}(z) C_{\Omega}\right\|$ which is based on the fact that $\Omega \subset G\left(I^{+}\right)$. Let $d_{\Omega}^{I^{+}} \equiv \inf _{x \in G\left(I^{+}\right)^{c}} d_{A}(x, \Omega)$; by construction of $\Omega, d_{\Omega}^{I^{+}} \geqq d_{12} / 2-\delta$.

Lemma 6.3. For $z \in \rho\left(H_{i . t}\right), i=1,2$, and $\operatorname{Re} z \leqq I^{+}$,

$$
\left\|C_{\Omega} R_{i}(z) C_{\Omega}\right\| \leqq 1+c e^{-2 d_{\Omega}^{I^{+}}}\left\|R_{i}(z)\right\|
$$

where the constant $c, 0<c<\infty$, is independent of $z, h$ and $F$.

Proof. For $i=2$, this result is proved in [BCD3]. For $i=1$, one easily repeats the proof of $[\mathrm{BCD} 3]$ using the fact that $H_{1, t}\left|C_{0}^{\infty}(\Omega)=H_{1}\right| C_{0}^{\infty}(\Omega)$.

Lemma 6.4. Let $\Gamma \subset \mathbb{C}$ be a simple closed contour passing through $I^{ \pm}$(see Fig. 6.1) and lying in $\mathcal{O}_{\beta}$ of Theorem 5.1. Let $\beta$ and $h$ be sufficiently small. Then $\|K(z)\|<1$ uniformly on $\Gamma$.

Proof. By Lemma 6.3, it suffices to estimate $\left\|R_{i}(z)\right\|$ along $\Gamma$. For $i=1$, it follows from part (2) of Theorem 5.1 that:

$$
\left\|R_{1}(z)\right\| \leqq c_{1}\left(\varepsilon_{0} \beta / 2-c_{2} h\right)^{-1} .
$$

By Lemma 2.2 and the spectral theorem

$$
\left\|R_{2}(z)\right\| \leqq c\left(F h^{2+\varepsilon}\right)^{-1} .
$$

Combining (6.26) and (6.27) with (6.25) and (6.22) we obtain

$$
\|K(z)\| \leqq 2 \delta^{-1}\left(1+\left(F h^{2+\varepsilon}\right)^{-1} e^{-2 d_{\Omega}^{I^{+}}}\right),
$$

so we must prove that there exists $0<\eta<1$ such that the right side of (6.28) is less than $\eta$. Now by (6.6) we can write $d_{\Omega}^{I^{+}}=h^{-1} \tilde{d}$, where $\tilde{d}$ is bounded below by a positive constant as $h \rightarrow 0$ so the second term on the right in (6.28) vanishes as $h \rightarrow 0$. Moreover, we can choose $\delta=d_{12} / 4=\tilde{d}_{12} / 4 h$, where $\tilde{d}_{12}$ is also bounded below as $h \rightarrow 0$, so it is clear that the right side of (6.28) can be made arbitrarily small by taking $h$ small.

Corollary 6.5. Let $\Gamma$ be as above. For all $h$ sufficiently small, $\Gamma \subset \rho\left(H_{t}\right), \operatorname{Im} t=\beta>0$ and small, and Eq. (6.20) holds on $\Gamma$.

We next estimate $\left\|P-J P_{0} \tilde{J}^{*}\right\|$, where $P$ is the Riesz projection for $H_{t}$ corresponding to $\Gamma$, which exists by Corollary 6.5 ,

$$
P=\frac{-1}{2 \pi i} \oint_{\Gamma} R_{t}(z) d z
$$

where $R_{t}(z) \equiv\left(z-H_{t}\right)^{-1}$, and $P_{0}$ is the corresponding projection for $H_{0, t}$ onto the spectral subspace associated with $\sigma\left(H_{0, t}\right) \cap\left[I^{-}, I^{+}\right]$. Note that $P_{0}=0 \oplus P_{2}$, where 
$P_{2}$ is the projector for $\mathrm{H}_{2}$ for the interval $\left[I^{-}, I^{+}\right]$. Recall that $M_{i}, i=1,2$, are supported in $G\left(I^{+}\right)$, and hence we will use semiclassical estimates on the resolvent localized to $G\left(I^{+}\right)$. We express $P-J P_{0} \tilde{J}^{*}$ as given on the right in (6.20) as the sum of four terms:

$$
\begin{aligned}
& I_{1}(z) \equiv J R_{0}(z) P_{0} M_{2}^{*}(1-K(z))^{-1} M_{1} P_{0} R_{0}(z) \tilde{J}^{*} \\
& I_{2}(z) \equiv J R_{0}(z) P_{0} M_{2}^{*}(1-K(z))^{-1} M_{1}\left(1-P_{0}\right) R_{0}(z) \tilde{J}^{*} \\
& I_{3}(z) \equiv J R_{0}(z)\left(1-P_{0}\right) M_{2}^{*}(1-K(z))^{-1} M_{1} P_{0} R_{0}(z) \tilde{J}^{*} \\
& I_{4}(z) \equiv J R_{0}(z)\left(1-P_{0}\right) M_{2}^{*}(1-K(z))^{-1} M_{1}\left(1-P_{0}\right) R_{0}(z) \tilde{J}^{*}
\end{aligned}
$$

By Lemma 6.4, $(1-K(z))^{-1}$ is uniformly bounded on $\Gamma$ for all $h$ sufficiently small.

Proposition 6.6. Let $0<F<\left\|v^{\prime}\right\|_{\infty}$ be fixed. Then for all $h$ sufficiently small and $\beta>0$ sufficiently small, there exists $k>0$ such that:

$$
\left\|P-J P_{0} \tilde{J}^{*}\right\| \leqq c h^{k}
$$

so $\sigma\left(H_{t}\right) \cap \mathcal{O}_{\beta} \neq \varnothing(\beta \equiv \operatorname{Im} t)$.

Proof. We first consider $I_{j}(z), j=1,2,3$, and express these terms using $C_{\Omega}$. From (6.24) and the fact that $\|J\| \leqq 1$, we have

$$
\begin{aligned}
\left\|J R_{0}(z) P_{0} M_{2}^{*}\right\| & \leqq \delta^{-1}\left\|C_{\Omega} R_{2}(z) P_{2}\right\| \\
\left\|J R_{0}(z)\left(1-P_{0}\right) M_{2}^{*}\right\| & \leqq \delta^{-1}\left\|C_{\Omega} R_{1}(z)\right\|+\delta^{-1}\left\|C_{\Omega}\left(1-P_{2}\right) R_{2}(z)\right\| \\
\left\|M_{1} P_{0} R_{0}(z) \tilde{J}^{*}\right\| & \leqq \delta^{-1}\left\|C_{\Omega} R_{2}(z) P_{2}\right\| \\
\left\|M_{1}\left(1-P_{0}\right) R_{0}(z) \tilde{J}^{*}\right\| & \leqq \delta^{-1}\left\|C_{\Omega} R_{1}(z)\right\|+\delta^{-1}\left\|C_{\Omega}\left(1-P_{2}\right) R_{2}(z)\right\| .
\end{aligned}
$$

As in [BCD3], it is easily shown that $D\left(H_{2}\right) \subset D\left(C_{\Omega}\right)$ so we have for some $c>0$, independent of $h$ and $F$ :

$$
\left\|C_{\Omega} P u\right\| \leqq c\left\|\chi_{\Omega} P_{2} u\right\|,
$$

where $\chi_{\Omega}$ is the characteristic function on $\Omega$. In [HN], it is proved that for any $N>0$, there exists $C_{N}>0$ such that

$$
\left\|\chi_{\Omega} P_{2}\right\| \leqq c_{N} h^{N} .
$$

In what follows, we will take $N$ large, so that $N \geq \frac{3}{2}(2+\varepsilon)$. (We remark that $\left\|\chi_{\Omega} P_{2}\right\|$ actually satisfies the bound $\left.\left\|\chi_{\Omega} P_{2}\right\| \leqq c e^{\left(d_{\Omega}^{I^{+}}-\varepsilon h^{-1}\right.}\right)^{2}$, see [BCD3].) To estimate $I_{1}(z)$ we write

$$
\begin{aligned}
\left|\oint_{\Gamma} I_{1}(z) d z\right| & \leqq c \oint_{\Gamma} d|z|\left\|C_{\Omega} P_{2}\right\|^{2}\left\|R_{2}(z)\right\|^{2} \delta^{-2} \\
& \leqq c|\Gamma| h^{2 N} h^{-2(2+\varepsilon)} \delta^{-2} .
\end{aligned}
$$

Since $|\Gamma|=\mathcal{O}\left(h^{2+\varepsilon}\right)$ and $\delta=\mathcal{O}(h)$, it follows that $\lim _{h \rightarrow 0} \oint_{\Gamma} I_{1}(z) d z=0$. Next, we consider $I_{2}$ and $I_{3}$ which are similar. Here we need an estimate of [BCD3] which is similar to Lemma 6.3:

$$
\left\|C_{\Omega} R_{i}(z)\right\| \leqq\left\|R_{i}(z)\right\|^{1 / 2}\left\{1+c e^{-2 d_{\Omega}^{I^{+}}}\left\|R_{i}(z)\right\|\right\}^{1 / 2} .
$$


Because of Lemma 2.2 and Theorem 5.1, we obtain the bounds

$$
\begin{gathered}
\left\|C_{\Omega} R_{1}(z)\right\| \leqq c, \\
\left\|C_{\Omega} R_{2}(z)\right\| \leqq c h^{-(1 / 2)(2+\varepsilon)},
\end{gathered}
$$

where we used the estimate on $d_{\Omega}^{I^{+}} \leqq c h^{-1}$. Considering $I_{2}(z)$ we write:

$$
\begin{aligned}
\left|\oint_{\Gamma} I_{2}(z) d z\right| & \leqq \delta^{-2} \oint_{\Gamma} d|z|\left\|C_{\Omega} P_{2}\right\|\left\|R_{2}(z)\right\|\left(\left\|C_{\Omega} R_{1}(z)\right\|+\left\|C_{\Omega^{2}} R_{2}(z)\right\|\right) \\
& \leqq c \delta^{-2}|\Gamma| h^{N} h^{-2-\varepsilon}\left(c_{1}+c_{2} h^{-(1 / 2)(\varepsilon+2)}\right),
\end{aligned}
$$

so by taking $N$ sufficiently large, this term also vanishes as $h \rightarrow 0$. The last integral, $I_{4}(z)$, requires special treatment. The operator $K(z)$ is decomposed into singular and non-singular parts according to the decomposition $R_{0}(z)=P_{0} R_{0}(z)+$ $\left(1-P_{0}\right) R_{0}(z)$. From (6.19), we write

$$
\begin{aligned}
K(z) & =M_{1} P_{0} R_{0}(z) M_{2}^{*}+M_{1}\left(1-P_{0}\right) R_{0}(z) M_{2}^{*} \\
& \equiv K_{s}(z)+K_{r}(z) .
\end{aligned}
$$

By the second resolvent identity, we obtain from (6.35):

$$
(1-K(z))^{-1}=\left(1-K_{r}(z)\right)^{-1}+(1-K(z))^{-1} K_{s}(z)\left(1-K_{r}(z)\right)^{-1}
$$

which is valid for $z \in \Gamma$ since $\left(1-K_{r}(z)\right)^{-1}$ is also uniformly bounded. In fact, it is holomorphic on and inside $\Gamma$. Consequently, its contribution to the contour integral of $I_{4}$ vanishes by Cauchy's theorem. Next, we estimate $K_{s}(z)$ using $C_{\Omega}$ via Lemma 6.2.:

$$
\begin{aligned}
\left\|K_{s}(z)\right\| & \leqq\left\|C_{\Omega} P_{2}\right\|\left\|C_{\Omega} R_{2}(z)\right\| \\
& \leqq c h^{N} h^{-(1 / 2)(2+\varepsilon)},
\end{aligned}
$$

where we used (6.30) and (6.34). Hence, the contour integral of $I_{4}(z)$ is bounded by:

$$
\begin{aligned}
\left|\oint_{\Gamma} I_{4}(z) d z\right| & \leqq \delta^{-2} \oint_{\Gamma} d|z|\left\|C_{\Omega} P_{2}\right\|\left\|C_{\Omega} R_{2}(z)\right\|\left(\left\|C_{\Omega} R_{1}(z)\right\|+\left\|C_{\Omega} R_{2}(z)\right\|\right)^{2} \\
& \leqq c h^{N} h^{-(1 / 2)(2+\varepsilon)}\left(c_{1}+c_{2} h^{-(1 / 2)(2+\varepsilon)}\right)^{2}
\end{aligned}
$$

which also vanishes as $h \rightarrow 0$. This completes the proof.

Theorem 6.7. Let $H(h, F)=h^{2} p^{2}+v+F x$, where $\left\|v^{\prime}\right\|_{\infty}>F>0$, and fixed. Then for $h$ sufficiently small, $H(h, F)$ has an infinite ladder of spectral resonances.

Proof. It follows easily from Proposition 6.6 using the decay properties of states in $\operatorname{Ran} P_{0}$ in the region $\Omega$ and to the left of $\Omega$, expressed by (6.30), that

$$
\operatorname{dim} \operatorname{Ran} P_{0}=\operatorname{dim} \operatorname{Ran} P,
$$

(see [BCD3] for a more detailed argument). Hence the total multiplicity of $\sigma\left(H_{t}\right)$ inside $\Gamma$ is at least one since $H_{2}$ has at least one eigenvalue $e_{0} \in I$ so $\operatorname{dim} \operatorname{Ran} P_{0} \geqq 1$.

Corollary 6.8. Let $z_{0} \in \sigma\left(H_{t}\right)$ lying inside $\Gamma$ as in Theorem 6.7. Let $S_{T}^{\prime}\left(I^{+}\right)$be the 
first turning point to the right of $S_{T}\left(I^{+}\right)$and set

$$
\rho_{T} \equiv \int_{S_{T}\left(I^{+}\right)}^{s_{T^{\prime}}^{\prime}\left(I^{+}\right)}\left(V(s)-I^{+}\right)_{+}^{1 / 2} d s .
$$

Then for any $\varepsilon>0$ there exists $h_{0}>0$ such that $h<h_{0}$ implies

$$
\left|\operatorname{Im} z_{0}\right| \leqq c e^{-2(1-\varepsilon) \rho T^{h-1}}
$$

for some $c>0$.

We remark that this estimate, which is an immediate result of the semiclassical theory of shape resonances, is basically insensitive to the field strength $F$ as $F \rightarrow 0$. We note, however, that it does give the Oppenheimer formula for the atomic Stark effect $[\mathrm{H}]$. To obtain an analogous result for the Stark ladder resonances, we must consider resonant and non-resonant wells which we do in the next section.

\section{Estimates on the Resonance Width}

In this section, we prove a generalization of an Oppenheimer-type estimate for the resonance width under certain conditions described below. More precisely, we show that for $F>0$ fixed and $h$ sufficiently small, there exists a constant $\alpha>0$ (independent of $h$ ) such that for some resonance eigenvalue $z_{0} \in \sigma\left(H_{t}\right)$,

$$
\left|\operatorname{Im} z_{0}\right| \leqq c e^{-\alpha / F} .
$$

For the Stark effect, the Oppenheimer formula for one electron atomis was proven by Herbst $[\mathrm{H}]$ and for $N$-electron atoms by Sigal [Si3].

The condition under which (7.1) is valid is expressed using the notion of resonant and non-resonant wells. This concept was used by Helffer and Sjöstrand [HSj3-4] and by Briet, Combes, and Duclos [BCD3] in their analysis of multiple-well tunneling.

Definition 7.1. Let $h_{k}$ be the single-cell Hamiltonian introduced in the proof of Lemma 2.2 and let $I \subset \mathbb{R}$ be a closed interval. A cell is said to be resonant for $I$ if $\sigma\left(h_{k}\right) \cap I \neq \varnothing$ and non-resonant otherwise.

We take $I$ to be the interval around $e_{0}$ of Sect. 6 so that

$$
\operatorname{dist}\left(\bigcup_{k} \sigma\left(h_{k}\right), I^{+}\right) \geqq c F h^{2+\varepsilon}
$$

for some $\varepsilon>0$. To prove estimate (7.1), we need two assumptions:

(A1) Ran $P$, where $P$ is the projection for $H_{t}$ and the interval $I$ defined in Sect. 6 , is spanned by eigenvectors of $H_{t}$ written as $\left\{\psi_{j}\right\}_{j=0}^{N_{0}}$.

(A2) Let $U_{0}$ be the farthest right unit cell with $\tilde{e}_{0} \in \sigma\left(h_{0}\right)$ and let $R$ be the set of all resonant cells for $I$. Then $d_{A}^{I^{+}}\left(U_{i}, U_{0}\right)=\mathcal{O}\left(F^{-1}\right)$ for any $i \in R, i \neq 0$, or if there is only one resonant cell $U_{0}$ for $I$, then $d_{A}^{I^{+}}\left(\Omega, U_{0}\right)=\mathcal{O}\left((h F)^{-1}\right)$, where $\Omega \equiv W_{1} \cap W_{2}$ is defined in Sect. 6. 
Remark 7.2.

1. We assume that $\left\{\psi_{j}\right\}_{j=0}^{N_{0}}$ is normalized as follows. Since $H_{t}^{*}=H_{t}$, we can take

$$
\left\langle\psi_{j}^{*}, \psi_{k}\right\rangle=\delta_{j k}
$$

where $\psi_{j}^{*}$, the complex conjugate of $\psi_{j}$, satisfies $H_{t}^{*} \psi_{j}^{*}=\bar{z}_{j} \psi_{j}^{*}$ if $H_{t} \psi_{j}=z_{j} \psi_{j}$.

2. (A1) excludes the accidental situation where $R_{t} \equiv\left(H_{t}-z\right)^{-1}$ has higher-order poles at the resonance eigenvalues.

3. The average spacing between the lowest eigenvalues of an individual cell Hamiltonian is $\mathcal{O}(h)$ by the harmonic approximation, if each cell contains a single, non-degenerate minimum. If $\operatorname{diam}(I)=\mathcal{O}\left(h^{N}\right)$, for some large $N$, as allowed by Lemma 2.2, then the fact that the one of the options in (A2) will hold depends critically on how small $h$ is with respect to $F$. If $F$ varies with $h$ and $F \geqq c h^{\alpha}$, $0<\alpha<1$, then the second option is generically satisfied, whereas for $F<h^{\alpha}, \alpha>1$, there are many resonant wells and the first options is generically obtained.

We now begin the proof of (7.1) assuming (A1) and (A2). Recall that $P_{2}$ is the projection for $\mathrm{H}_{2}$ on the interval $I$. We label the unit cells right to left $U_{0}, U_{1}, \ldots, U_{N-1}$, so $U_{N-1}$ is adjacent and to the right of $S_{T}\left(e_{0}\right)$. Let $U_{R_{1}}$ be the first resonant cell for $I$ to the left of $U_{0}$. We introduce some cut-off functions, see Fig. 7.1. Let $\eta_{R} \in C^{1}, 0 \leqq \eta_{R} \leqq 1$, with $\eta_{R} \mid\left(-\infty,-R_{1} \tau-\varepsilon\right]=1$ for some $\varepsilon>0$ to be fixed below, and $\operatorname{supp} \eta_{R}^{\prime} \subset G\left(I^{+}\right)$. Let $J_{R} \in C^{1}, 0 \leqq J_{R} \leqq 1$, with $J_{R} \mid\left[-R_{1} \tau+\varepsilon, \infty\right)=1$ and $\eta_{R} J_{R}=0$. In the case that $U_{0}$ is the only resonant well, we take $\eta_{R}\left|\left(W_{1} \backslash \Omega\right)=1, J_{R}\right|\left(W_{2} \backslash \Omega\right)=1$, in such a way that $\eta_{R} J_{R}=0$. Finally, let $\eta_{0} \in C^{1}, 0 \leqq \eta_{0} \leqq 1$, with $\eta_{0} \mid[-\tau+\varepsilon, \infty)=1$ and $J_{R} \eta_{0}=\eta_{0}$.

We define approximate resonant wave functions as follows. Let $\phi_{k}, k=0$, $R_{1}, \ldots, R_{N_{0}}$ be real eigenfunctions of $h_{R_{k}}\left(R_{0}=0\right)$ with eigenvalues $e_{k} \in I$ and $\left\|\phi_{k}\right\|=1$. Define

$$
\begin{aligned}
& \Phi_{k} \equiv P \eta_{R} \phi_{k}, \quad k=R_{1}, \ldots, R_{N_{0}} \\
& \Phi_{0} \equiv P \eta_{0} \phi_{0} .
\end{aligned}
$$

Lemma 7.3. For $h$ small enough, $\left\{\Phi_{k}, k=0, R_{1}, \ldots, R_{N_{0}}\right\}$ forms a basis for $\operatorname{Ran} P$.

Proof. We first construct a basis for $\operatorname{Ran} P_{2}$. Let $\tilde{\Phi}_{0}=P_{2} \eta_{0} \phi_{0}$ and $\tilde{\Phi}_{k}=P_{2} \eta_{R} \phi_{k}$ for $k>0$. It then follows from general stability results for tunneling (see, for example [BCD3]) that for $h$ small enough the set $\left\{\widetilde{\Phi}_{k}, k=0, R_{1}, \ldots, R_{N_{0}}\right\}$ is a basis for $\operatorname{Ran} P_{2}$ and there exists $a>0$ that

$$
\left\langle\tilde{\Phi}_{j}, \tilde{\Phi}_{j}\right\rangle=\delta_{i j}+\mathcal{O}\left(e^{-a h^{-1}}\right) \text {. }
$$

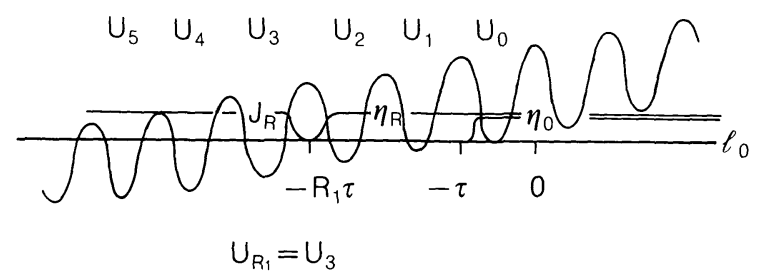

Fig. 7.1. Cut-off functions $\eta_{R}$ and $J_{R}$ and the wells $U_{i}$ 
Defining $\eta_{j}=\eta_{R}$ if $j>0$, we express $\Phi_{j}$ as

$$
\Phi_{j}=\left(P-P_{2}\right) \eta_{j} \phi_{j}+\tilde{\Phi}_{j}
$$

By Proposition 6.6 and the fact that states in $\operatorname{Ran} P_{2}$ are exponentially small in $G\left(I^{+}\right)$(see (6.33) and the discussion there), we obtain

$$
\Phi_{j}=\widetilde{\Phi}_{j}+\mathcal{O}\left(h^{k}\right)
$$

for some $k>0$. The proof now follows easily from this.

Proposition 7.4. Let $\bar{S}_{0} \equiv \min _{k=1, \ldots, N_{0}}\left\{d_{A}^{I^{+}}\left(U_{0}, U_{R_{k}}\right), d_{A}^{I^{+}}\left(U_{0}, \Omega\right)\right\}$. Then for any $\delta>0$ and $\kappa, 0<\kappa<1$, we have for $h$ small enough,

$$
\text { (i) }\left|\left\langle\Phi_{0}^{*}, H_{t}^{\alpha} \Phi_{k}\right\rangle\right| \leqq e^{-\left(\kappa \bar{S}_{0}-\delta R_{1}\right)} \text {, }
$$

for $\alpha=0,1$,

$$
\text { (ii) }\left|\operatorname{Im}\left\langle\Phi_{0}^{*}, H_{t} \Phi_{0}\right\rangle\right| e^{-\left(\kappa \bar{S}_{0}-\delta R_{1}\right)} \text {. }
$$

Proof. Let $\tilde{H}=h^{2} p^{2}+\tilde{V}$, where $\tilde{V} \mid \operatorname{supp} J_{R}=V$ and $\lim _{x \rightarrow-\infty} \tilde{V}=\infty$ with $\tilde{V}=\mathcal{O}(|x|)$ and monotone. The geometric resolvent equation for $R_{t}$ and $\tilde{R}$ is

$$
J_{R} R_{t}=\tilde{R} J_{R}+\tilde{R} W\left(J_{R}\right) R_{t},
$$

where $W\left(J_{R}\right)=h^{2}\left[p^{2}, J_{R}\right]$. Let $\Gamma$ be the contour through $I^{+}$as in Lemma 6.4. Then,

$$
\left\langle\Phi_{0}^{*}, H_{t}^{\alpha} \Phi_{k}\right\rangle=\frac{1}{2 \pi i} \oint_{\Gamma} d z z^{\alpha}\left\langle\eta_{0} \phi_{0}, \tilde{R}(z) W\left(J_{R}\right) R_{t}(z) \eta_{R} \phi_{k}\right\rangle,
$$

since $J_{R} \eta_{R}=0$ and $J_{R} \eta_{0}=\eta_{0}$ by construction, and

$$
\operatorname{Im}\left\langle\Phi_{0}^{*}, H_{t} \Phi_{0}\right\rangle=\frac{1}{2 \pi i} \oint_{\Gamma} d z z\left\langle\eta_{0} \phi_{0}, \tilde{R}(z) W\left(J_{R}\right) R_{t}(z) \eta_{0} \phi_{0}\right\rangle .
$$

Here we used the fact that $J_{R} \eta_{0}=\eta_{0}$ and that

$$
\operatorname{Im}\left[(2 \pi i)^{-1} \oint_{\Gamma} d z z\left\langle\eta_{0} \phi_{0}, \tilde{R}(z) \eta_{0} \phi_{0}\right\rangle\right]=\operatorname{Im}\left\langle\eta_{0} \phi_{0}, \tilde{H} \tilde{P}(I) \eta_{0} \phi_{0}\right\rangle=0,
$$

where $\tilde{P}(I)$ is the spectral projector for $\tilde{H}$ and $I$. It remains to estimate $\left\|\tilde{R}(z) W\left(J_{R}\right) R_{t}(z) \eta_{0}\right\|$ uniformly on $\Gamma$ which we do in a series of lemmas below.

It will be convenient to estimate the adjoint quantity on $\Gamma$, i.e. $R_{\bar{t}}(z) W\left(J_{R}\right) \tilde{R}(z) \eta_{0}$, which follows since we can take $\Gamma$ symmetric under complex conjugation.

Lemma 7.5. Let $\Omega_{R} \equiv \operatorname{supp} J_{R}^{\prime}$. Then for any $\delta>0$ and $\kappa, 0<\kappa<1$, we have for $h$ sufficiently small,

$$
\left\|C_{\Omega_{R}} \tilde{R}(z) \eta_{0}\right\| \leqq e^{-\left(\kappa \bar{S}_{0}-\delta R_{1}\right)}
$$

uniformly on $\Gamma$, where $C_{\Omega_{R}}$ is defined in (6.21).

Proof. Let $\mathcal{O}=\operatorname{supp}\left(V-I^{+}\right)_{-}, \mathcal{O}_{k} \equiv U_{k} \cap \mathcal{O}$, and $d_{k} \equiv \inf _{j \neq k} d_{A}^{I^{+}}\left(\mathcal{O}_{k}, \mathcal{O}_{j}\right)$ (see Fig. 7.2). For any $\delta>0$, one has $\delta<\frac{1}{2} d_{k}, k=1, \ldots, R_{1}$, for $h$ sufficiently small. We define 


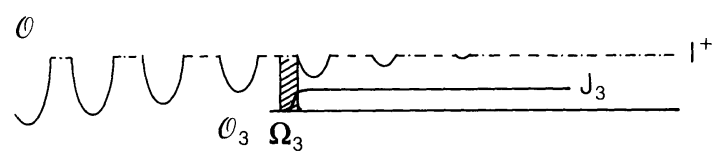

Fig. 7.2. Localization regions for resonant wells

for $k=1, \ldots, R_{1}, J_{k} \in C^{1}, 0 \leqq J_{k} \leqq 1$, such that $\Omega_{k} \equiv \operatorname{supp} J_{k}^{\prime}$ satisfies

(i) $d_{A}^{I^{+}}\left(\Omega_{k}, \mathcal{O}_{k}\right)=d_{A}^{I^{+}}\left(\Omega_{k}, \mathcal{O}_{k-1}\right)$.

(ii) $\Omega_{k}$ has diameter $\delta$.

Then $J_{k}$ is chosen to be equal to 1 (respectively, 0) to the right (respectively, left) of $\Omega_{k}$. Furthermore, we can choose $J_{k}$ such that an inequality like (6.11) holds. Recalling the definitions of $J_{R}$ and $\eta_{0}$ above, we choose $\varepsilon$ there so that $J_{R}=J_{R_{1}}$ and $J_{1}=\eta_{0}$.

Now let $\tilde{H}_{k}$ be the local Hamiltonian $\tilde{H}_{k}=h^{2} p^{2}+\tilde{V}_{k}$, where $\tilde{V}_{k} \mid \operatorname{supp} J_{k}=V$ and $\widetilde{V}_{k}$ grows monotonically as $x \rightarrow-\infty$ like $\mathcal{O}(|x|)$ outside the supp $J_{k}$. We take $\tilde{H}_{R_{1}}=\widetilde{H}$ as in Proposition 7.3. We then have the following identity:

$$
C_{\Omega_{R}} \tilde{R}(z) \eta_{0}=C_{\Omega_{R}} \tilde{R}(z) W_{R_{1}-1} \tilde{R}_{R_{1}-1}(z) W_{R_{1}-2} \cdots W_{1} \tilde{R}_{1}(z) \eta_{0},
$$

where $W_{k}=-h^{2}\left[p^{2}, J_{k}\right]$. This is obtained by iterating the geometric resolvent equation. For $k=R_{1}-1$,

$$
\tilde{R}(z) J_{R_{1}-1}=J_{R_{1}-1} \tilde{R}_{R_{1}-1}(z)+\tilde{R}(z) W_{R_{1}-1} \tilde{R}_{R_{1}-1}(z) .
$$

Since we have constructed the cut-off functions such that $J_{R_{1}-1}=0$ on $\Omega_{R}=\operatorname{supp} J_{R_{1}}^{\prime}$ and $J_{R_{1}-1}=1$ on $\operatorname{supp} \eta_{0}$, we obtain

$$
C_{\Omega_{R}} \tilde{R}(z) \eta_{0}=C_{\Omega_{R}} \tilde{R}(z) W_{R_{1}-1}(z) \eta_{0} .
$$

Equation (7.9) now follows by repeated application of this procedure.

Finally, to obtain (7.8), we use the factorization trick (see Appendix 2) to obtain from (7.9),

$$
\left\|C_{\Omega_{R}} \tilde{R}(z) \eta_{0}\right\| \leqq \delta^{-R_{1} / 2}\left(\prod_{k=1}^{R_{1}-1}\left\|C_{\Omega_{k+1}} \tilde{R}_{k}(z) C_{\Omega_{k}}\right\|\right)\left\|C_{\Omega_{1}} \tilde{R}(z) \eta_{0}\right\| .
$$

We use Theorem A1.3 of Appendix 1 to estimate the product

$$
\begin{aligned}
\prod_{k=1}^{R_{1}-1}\left\|C_{\Omega_{k+1}} \tilde{R}_{k}(z) C_{\Omega_{k}}\right\| & \leqq\left(\prod_{k=1}^{R_{1}-1}\left\|\tilde{R}_{k}(z)\right\|\right) e^{-\left(\bar{S}_{0}-\delta R_{1}\right)} \\
& \leqq e^{-\left(\kappa \bar{S}_{0}-\delta R_{1}\right)}
\end{aligned}
$$

for some $0<\kappa<1$ and $h$ small enough. Here we used the non-resonant condition as expressed through bounds of the type (6.27) and we used the fact that $\bar{S}_{0}=\mathcal{O}\left(h^{-1}\right)$. By (6.32), the last factor on the right in (7.10) only changes the factors $\kappa$ in this estimate.

Lemma 7.6. For any $\delta>0$ and $\kappa, 0<\kappa<1$, we have

$$
\left\|R_{i}(z) W\left(J_{R}\right) R(z) \eta_{0}\right\| \leqq e^{-\left(\kappa \bar{S}_{0}-\delta R_{1}\right)},
$$

for $h$ small enough, uniformly on $\Gamma$. 
Proof. We again the factorization trick with respect to $\Omega_{R}$ to obtain

$$
\left\|R_{\bar{t}}(z) W\left(J_{R}\right) \tilde{R}(z) \eta_{0}\right\| \leqq\left\|C_{\Omega_{R}} R_{\bar{t}}(z)\right\|\left\|C_{\Omega_{R}} \tilde{R}(z) \eta_{0}\right\| .
$$

By (6.32) and the fact that $\left\|R_{t}(z)\right\|$ and, consequently, $\left\|R_{i}(z)\right\|$, is at most polynomially large in $h^{-1}$ on $\Gamma$ by Lemma 6.4 and (6.20), the conclusion follows from (7.8)

We are now ready to prove our main result.

Theorem 7.7. Under condition (A1), there exists a constant $\kappa, 0<\kappa<1$ such that for $h$ small enough, $H(h, F)$ has at least one ladder of spectral resonances with

$$
\left|\operatorname{Im} z_{0}\right| \leqq e^{-\kappa \bar{S}_{0}},
$$

where $\bar{S}_{0}=\mathcal{O}\left(h^{-1}\right)$. In particular, if (A2) is also satisfied, then there exists a constant $\alpha>0$ such that for all $h$ sufficiently small

$$
\left|\operatorname{Im} z_{0}\right| \leqq e^{-\alpha / \boldsymbol{F}} .
$$

Proof. We use the Gram-Schmidt procedure to construct an orthonormal set of approximate resonance wave functions from $\left\{\Phi_{k}, k=0, \ldots, R_{N_{0}}\right\}$ defined in (7.3). We arrange it so that the new functions denoted by the same symbol $\Phi_{k}$, satisfy (i) $\Phi_{0}$ is constructed from the old $\Phi_{0}$, and (ii)

$$
\left\langle\Phi_{j}^{*}, \Phi_{k}\right\rangle=\delta_{j k} \quad\left(\text { where } \Phi_{k}^{*}=\bar{\Phi}_{k}\right) .
$$

It follows easily from (7.4) that this new system still satisfies Proposition 7.4. In the verification of this we use the fact that $\left\langle\Phi_{i}^{*}, \Phi_{j}\right\rangle$ is bounded by 1 . Recall that $\left\{\psi_{j}\right\}_{j=0}^{N_{0}}$ are the true eigenfunctions of $H_{t}$ which span $\operatorname{Ran} P$ by (A1) and satisfy (7.2). Since $\Phi_{0} \in \operatorname{Ran} P$, we have

$$
\left\langle\Phi_{0}^{*}, \Phi_{0}\right\rangle=\sum_{k=0}^{N_{0}}\left|\left\langle\psi_{k}^{*}, \Phi_{0}\right\rangle\right|^{2}=1 .
$$

Since $N_{0} \leqq N=\mathcal{O}\left(F^{-1}\right)$ and independent of $h$ by (2.2), there exists at least one $\psi_{k}$, say $\psi_{0}$, such that

$$
\left|\left\langle\psi_{0}^{*}, \Phi_{0}\right\rangle\right|^{2} \geqq c F,
$$

for some constant $c>0$. Let $H_{t} \psi_{0}=z_{0} \psi_{0}$. Then we obtain

$$
\left|\operatorname{Im} z_{0}\right|=\left|\operatorname{Im} \frac{\left\langle\Phi_{0}^{*}, H_{t} \psi_{0}\right\rangle}{\left\langle\Phi_{0}^{*}, \psi_{0}\right\rangle}\right| .
$$

Upon expanding $\psi_{0}$ in the basis $\left\{\boldsymbol{\Phi}_{k}\right\}_{k=1}^{N_{0}}$ as

$$
\psi_{0}=\sum_{k=0}^{N_{0}}\left\langle\Phi_{k}^{*}, \psi_{0}\right\rangle \Phi_{k}
$$

and inserting this into the numerator on the right side of (7.12), we obtain

$$
\left|\operatorname{Im} z_{0}\right| \leqq\left|\operatorname{Im}\left\langle\Phi_{0}^{*}, H_{t} \Phi_{0}\right\rangle\right|+c F^{-3 / 2}\left(\max _{k=1, \ldots, N_{0}}\left|\left\langle\Phi_{0}^{*}, H_{t} \Phi_{k}\right\rangle\right|\right),
$$

where we used (2.2), (7.11) and the fact that $\left|\left\langle\Phi_{k}^{*}, \psi_{0}\right\rangle\right| \leqq 1$, for all $k$. The proof then follows immediately from Proposition 7.3 


\section{Appendix 1. Localized Resolvent Estimates}

We sketch the proofs, for the convenience of the reader, of the resolvent estimates in the classically forbidden region $G\left(I^{+}\right)$used in this paper. Some of these estimates appear already in [HeSj] and [BCD3]. They are based on the inequality (6.9) for the Agmon distance and the identity

$$
\|\nabla(\xi v)\|^{2}=\operatorname{Re}\left\langle\xi^{2} v,-\Delta v\right\rangle+\||\nabla \xi| v\|^{2}
$$

valid for all $v \in H^{2}\left(\mathbb{R}^{n}\right)$ and may a.e. differentiable function $\xi$.

Consider first the Hamiltonian $H^{(0)}=h^{2} p^{2}+V^{(0)}$, where $V^{(0)} \equiv \sup \left(V, I^{+}\right)$, i.e. $V^{(0)}$ is obtained from $V$ by filling-up all the wells to energy $I^{+}$. We assume that $\sigma\left(H^{(0)}\right)$ and $\sigma(H), H=h^{2} p^{2}+V$, are discrete near $I^{+}$and dist $\left(\sigma\left(H^{(0)}\right), I^{+}\right)=\mathcal{O}\left(h^{k}\right)$, some $k>0$, and similarly for $H$.

Lemma A1.1. For all $z$ with $R e z \leqq I^{+}$,

$$
\left\|C_{\Omega} R^{(0)}(z) C_{\Omega}\right\| \leqq 1 .
$$

Proof. By the definition of $C_{\Omega}$ in (6.21) and the positivity of $\left(V^{(0)}-I^{+}\right)$, we have for any $v \in H^{1}(\Omega)$,

$$
\begin{aligned}
\left\|C_{\Omega} v\right\|^{2} & \leqq\left\langle v,\left[h^{2} p^{2}+\left(V^{(0)}-I^{+}\right)\right] v\right\rangle \\
& \leqq \operatorname{Re}\left\langle v,\left(H^{(0)}-z\right) v\right\rangle .
\end{aligned}
$$

We take $v==R^{(0)}(z) C_{\Omega} u,\|u\|=1$ and $u \in H^{1}(\Omega)$, and obtain

$$
\left\|C_{\Omega} R^{(0)}(z) C_{\Omega} u\right\|^{2} \leqq\left\|C_{\Omega} R^{(0)}(z) C_{\Omega} u\right\|
$$

Lemma A1.2. Suppose $\Omega_{1}, \Omega_{2} \subset G\left(I^{+}\right)$and that $d_{A}^{I^{+}}\left(\Omega_{1}, \Omega_{2}\right)>0$. Then for any $z$ with $\operatorname{Re} z \leqq I^{+}$,

$$
\left\|C_{\Omega_{1}} R^{(0)}(z) C_{\Omega_{2}}\right\| \leqq e^{-d_{A}^{I^{+}}\left(\Omega_{1}, \Omega_{2}\right)} .
$$

Proof. Let $\rho_{1}(x)=\inf \left(d_{A}^{I^{+}}\left(x, \Omega_{1}\right), d_{A}^{I^{+}}\left(\Omega_{1}, \Omega_{2}\right)\right)$ so that

$$
\begin{aligned}
& \left|\nabla\left(e^{-\rho_{1}}\right)\right|^{2} \leqq h^{-2}\left(V^{(0)}-I^{+}\right) e^{-2 \rho_{1}} \quad \text { on } \quad \mathbb{R} \backslash\left(\Omega_{1} \cup \Omega_{2}\right) \\
& =0 \quad \text { on } \Omega_{1} \cup \Omega_{2} \text {. }
\end{aligned}
$$

Then for all $v \in H^{1}(\Omega)$, it follows from (A.1), (6.21) and the above fact that

$$
\begin{aligned}
\left\|C_{\Omega_{1}} v\right\|^{2}= & \left\|C_{\Omega_{1}}\left(e^{-\rho_{1}} v\right)\right\|^{2} \\
\leqq & h^{2}\left\|\nabla\left(e^{-\rho_{1}} v\right)\right\|^{2}+\left\langle e^{-2 \rho_{1}} v,\left(V^{(0)}-I^{+}\right) v\right\rangle_{\Omega_{1}} \\
\leqq & \operatorname{Re}\left\langle e^{-2 \rho_{1}} v,-h^{2} \Delta v\right\rangle+\left\langle e^{-2 \rho_{1}} v,\left(V^{(0)}-I^{+}\right) v\right\rangle_{\Omega_{1}} \\
& +\left\langle e^{-2 \rho_{1}} v,\left(V^{(0)}-I^{+}\right) v\right\rangle_{R \backslash \Omega_{1} \cup \Omega_{2}}+\left\langle e^{-2 \rho_{1}} v,\left(V^{(0)}-I^{+}\right) v\right\rangle_{\Omega_{2}} \\
\leqq & \operatorname{Re}\left\langle e^{-2 \rho_{1}} v,\left(H^{(0)}-z\right) v\right\rangle .
\end{aligned}
$$

Here, $\langle w, u\rangle_{\Omega}$ denotes $\int_{\Omega} w^{*} u$. As above, we choose $v=R^{(0)}(z) C_{\Omega_{2}} u$, for $\|u\|=1$ and $u \in H^{1}\left(\Omega_{2}\right)$, and obtain, from (A.2),

$$
\left\|C_{\Omega_{1}} R^{(0)}(z) C_{\Omega_{2}} u\right\|^{2} \leqq \operatorname{Re}\left\langle R^{(0)}(z) C_{\Omega_{2}} u, e^{-2} \rho^{1} C_{\Omega_{2}} u\right\rangle .
$$


Since $\rho_{1}=d_{A}^{I^{+}}\left(\Omega_{1}, \Omega_{2}\right)$ on $\Omega_{2}$,

$$
\begin{aligned}
\left\|C_{\Omega_{1}} R^{(0)}(z) C_{\Omega_{2}} u\right\|^{2} & \leqq e^{-2 d_{A}^{I^{+}\left(\Omega_{1}, \Omega_{2}\right)}\left\|C_{\Omega_{2}} R^{(0)}(z) C_{\Omega_{2}}\right\|} \\
& \leqq e^{-2 d_{A}^{I^{+}\left(\Omega_{1}, \Omega_{2}\right)}}
\end{aligned}
$$

by Lemma A1.1.

Theorem A1.3. Let $U \equiv \operatorname{supp}\left(V-I^{+}\right)_{-}$, the union of wells, and let $\Omega_{i} \subset G\left(I^{+}\right)$be such that $d_{A}^{I^{+}}\left(\Omega_{i}, U\right)>\delta, i=1,2$, for some $\delta>0$. Then for any $z, \operatorname{Re} z \leqq I^{+}, \operatorname{Im} z \neq 0$ :

$$
\left\|C_{\Omega_{1}} R(z) C_{\Omega_{2}}\right\| \leqq e^{-d_{A}^{I^{+}}\left(\Omega_{1}, \Omega_{2}\right)}+c e^{-\left[d_{A}^{I^{+}}\left(\Omega_{1}, U\right)+d_{A}^{I^{+}}\left(\Omega_{2}, U\right)\right]}\|R(z)\|,
$$

where $c>0$ is a constant depending only on $\delta$.

Proof. We define two cut-off functions as follows. Let $j \in C^{1}, 0 \leqq j \leqq 1$, with $j(0)=0$, $j(\rho)=1$ if $\rho>\delta / 2$. Define $J(x) \equiv j\left(d_{A}^{I^{+}}(x, U)\right)$. Next, let $\tilde{j} \in C^{1}, 0 \leqq \tilde{j} \leqq 1$, with $\tilde{j}(\rho)=0$ if $\rho<\delta / 2$ and $\tilde{j}(\rho)=1$ if $\rho>\delta$. Define $\widetilde{J}(x) \equiv \tilde{j}\left(d_{A}^{I^{+}}(x, U)\right)$. Then, with $W(J) \equiv h^{2}\left[p^{2}, J\right]$, we have $W(J) \tilde{J}=0$. The geometric resolvent equation for $R$ and $R^{(0)}$ gives

$$
R(z) J=J R^{(0)}(z)-R(z) W(J) R^{(0)}(z) .
$$

Similarly, using $\tilde{J}$, we obtain

$$
\tilde{J} R(z)=R^{(0)}(z) \tilde{J}+R^{(0)}(z) W(\tilde{J}) R(z) .
$$

Upon multiplying (A.4) on the left by $\widetilde{J}$ and substituting (A.5) into the second term on the right of (A.4), we obtain

$$
\tilde{J} R(z) J=\tilde{J} R^{(0)}(z)-R^{(0)}(z) W(\tilde{J}) R(z) W(J) R^{(0)}(z) .
$$

Recalling that $J, \tilde{J} \mid \Omega_{i}=1, i=1,2$, it follows from (A.6) that

$$
C_{\Omega_{1}} R(z) C_{\Omega_{2}}=C_{\Omega_{1}} R^{(0)}(z) C_{\Omega_{2}}-C_{\Omega_{1}} R^{(0)}(z) W(\tilde{J}) R(z) W(J) R^{(0)}(z) C_{\Omega_{2}} .
$$

To estimate (A.7), we use Lemma A1.2 and Theorem A2.1 below. This gives the result using the fact that $d_{A}^{I^{+}}\left(\Omega_{1}\right.$, supp $\left.\widetilde{J}^{\prime}\right)=d_{A}^{I^{+}}\left(\Omega_{1}, U\right)-\delta / 2$. A similar estimate is obtained for $d_{A}^{I^{+}}\left(\Omega_{2}\right.$, supp $\left.J^{\prime}\right)$ in terms of $d_{A}^{I^{+}}\left(\Omega_{2}, U\right)$.

We also note the following result proved in [BCD3].

Proposition A1.4. Let $\Omega=\Omega_{1} \subset G\left(I^{+}\right)$and $U$ be as in Theorem A1.3. Then if $\operatorname{Re} z \leqq I^{+}$,

Corollary A1.4. Let $\Omega=\Omega_{1} \subset G\left(I^{+}\right)$and $U$ be as in Theorem A1.3. Then if $\operatorname{Re} z \leqq I^{+}$,

$$
\left\|C_{\Omega} R(z) C_{\Omega}\right\| \leqq 1+c e^{-2 d_{A}^{+}(\Omega, U)}\|R(z)\| .
$$

In Sect. 7, we apply Theorem A1.3 to the local Hamiltonians $\tilde{R}_{k}(z)$ whose spectrum is discrete and dist $\left(\sigma\left(\tilde{H}_{k}\right), I^{+}\right) \geqq c h^{2+\varepsilon}$, some $\varepsilon>0$. Moreover, we apply Proposition A1.4 in Lemma 6.3 to $R_{i}(z), i=1,2$. For $R_{2}(z)$, the hypotheses are satisfied in view of Lemma 2.2. For $R_{1}(z)$, we note that (A.6) and (A.7) hold with $\Omega_{1}=\Omega_{2}=\Omega$ and $R^{(0)}(z)=\left(h^{2} p^{2}+\widetilde{V}^{(0)}-z\right)$, where $\tilde{V}^{(0)} \mid W_{2}=V^{(0)}$ and $V \rightarrow \infty$ as $x \rightarrow-\infty$. This is because supp $\tilde{J}$ and $\operatorname{supp} J$ lie in the region where the vector field $f$ vanishes and consequently $H_{t} \mid \operatorname{supp} J=\left(h^{2} p^{2}+V\right)$. 


\section{Appendix 2. The Factorization Trick}

Geometric perturbation theory as used, for example, in Sect. 6, involves first order differential operators of the form

$$
W(J)=h^{2}\left[-\frac{d^{2}}{d x^{2}}, J\right]
$$

with $\Omega \equiv \operatorname{supp} J^{\prime} \subset G\left(I^{+}\right)$. Some typical functions $J$ which we consider have the following form. Given $\mathcal{O} \subset \mathbb{R}$, let $J(x)=j\left(d_{A}^{I^{+}}(x, \Omega)\right)$ where i) $j$ is piecewise linear, ii) $j(0)=1$, iii) $j(\rho)=0$, if $\rho>\delta$ for some $\delta>0$. In particular, if $\Omega=\left\{x \mid 0<d_{A}^{I^{+}}(x, \mathcal{O})<\delta\right\}$, then a) supp $J^{\prime} \subset \Omega$, and, b) $\left|J^{\prime}(x)\right| \leqq(h \delta)^{-1}\left(V(x)-I^{+}\right)_{+}^{1 / 2}$ a.e. on $\Omega$ and zero elsewhere.

The factorization trick [BCD3] consists in introducing the following operators from $H^{1}(\mathbb{R})$ to $L^{2}(\mathbb{R}) \oplus L^{2}(\mathbb{R})$

$$
\begin{aligned}
& M_{1}(J) u=h \delta^{1 / 2} J^{\prime} u \oplus h \delta^{-1 / 2} \chi_{\Omega} u^{\prime}, \\
& M_{2}(J) v=h \delta^{-1 / 2} \chi_{\Omega} v^{\prime} \oplus h \delta^{1 / 2} J^{\prime} v,
\end{aligned}
$$

where $\chi_{\Omega}$ is the characteristic function on $\Omega$. The following statement is easily proved.

Theorem A2.1.

1. $W(J)=M_{2}^{*}(J) M_{1}(J)$.

2. $\left\|M_{i}(J) u\right\| \leqq \delta^{-1 / 2}\left\|C_{\Omega} u\right\|, i=1,2$, for all $u \in H^{1}(\mathbb{R})$, where $C_{\Omega}$ is defined in

\section{References}

[AF] Agler, J., Froese, R.: Existence of Stark Ladder resonances. Commun. Math. Phys. 100, 161-171 (1985)

[AC] Aguilar, J., Combes, J.-M.: A class of analytic perturbations for one-body Schrödinger Hamiltonians. Commun. Math. Phys. 22, 269-279 (1971)

[A1] Avron, J. E.: The lifetime of Wannier ladder states. Ann. Phys. 143, 33-53 (1982)

[A2] Avron, J. E.: Binding of one-dimensional Bloch electrons by external fields. Phys. Rev. B16, 711-713 (1977)

[AZ] Avron, J. E., Zak, J.: Stability of band structure for external fields. Phys. Rev. B9, 658-663 (1974)

[BC] Balslev, E., Combes, J.-M.: Spectral properties of many-body Schrödinger operators with dilation analytic potentials. Commun. Math. Phys. 22, 280-294 (1971)

[B] Bentosela, F.: Bloch electrons in constant electric field. Commun. Math. Phys. 68, 173-182 (1979)

[BCDSSW] Bentosela, F., Carmona, R., Duclos, P., Simon, B., Souillard, B., Weder, R.: Schrödinger operators with an electric field and random or deterministic potentials. Commun. Math. Phys. 88, 387-397 (1983)

[BCD1] Briet, Ph., Combes, J.-M., Duclos, P.: On the location of resonances for Schrödinger operators in the semi-classical limit I. Resonance free domains. J. Math. Anal. Appl. 126, 90-99 (1987)

[BCD2] Briet, Ph., Combes, J.-M., Duclos, P.: On the location of resonances for Schrödinger operators II. Barrier top resonances. Commun. Partial Diff. Equations 12, 201-222 (1987) 
[BCD3] Briet, Ph., Combes, J.-M., Duclos, P.: Spectral stability under tunnelling. Commun. Math. Phys. 126, 133-156 (1989)

[BCD4] Briet, Ph., Combes, J.-M., Duclos, P., in preparation

[BD1] Buslaev, V. S., Dmitrieva, L. A.: Spectral properties of the Bloch electrons in external fields. University of California, Berkeley preprint PAM-477, 1989

[BD2] Buslaev, V. S., Dmitrieva, L. A.: Adiabatic perturbation of a periodic potential II. Teor. Mat. Fiz. 73, 430-442 (1987)

[BG] Bentosela, F., Grecchi, V.: Stark Wannier Ladders. Marseille preprint, 1989, to appear in Commun. Math. Phys.

[BGZ] Bentosela, F., Grecchi, V., Zironi, F.: Oscillations of Wannier Resonances. Phys. Rev. Letts. 50, 84-86 (1983)

[CDKS] Combes, J.-M., Duclos, P., Klein, M., Seiler, R.: The shape resonance. Commun. Math. Phys. 110, 215-236 (1987)

[CDS] Combes, J.-M., Duclos, P., Seiler, R.: Krein's formula and one-dimensional multiple well. J. Funct. Analysis 52, 257-301 (1983)

[CFKS] Cycon, H., Froese, R., Kirsch, W., Simon, B.: Schrödinger Operators, with Applications to Quantum Mechanics and Global Geometry. Texts and Monographs in Physics. Berlin, Heidelberg, New York: Springer 1987

[CH1] Combes, J.-M., Hislop, P. D.: Quantum tunnelling, non-trapping, and the existence of Stark Ladder resonances. J. Phys. A: Math. Gen. 23, 1501-1511 (1990)

[CH2] Combes, J.-M., Hislop, P. D.: Quantum tunnelling for Bloch electrons in small electric fields. In: Recent Developments in Quantum Mechanics, A. M. Boutet de Montvel (ed.). Kluger: Academic Publishing 1991

[DeBH] DeBièvre, S., Hislop, P. D.: Spectral resonances for the Laplace-Beltrami operator. Ann. Inst. Henri Poincare 48, 105-146 (1988)

[HSj1] Helffer, B., Sjöstrand, J.: Resonances en limite semi-classique. Suppl. Bull. S.M.F. 114 (1986)

[HSj2] Helffer, B., Sjöstrand, J.: Multiple wells in the semi-classical limit I. Commun. Partial Differ. Equations 9, 337-408 (1984)

[HSj3] Helffer, B., Sjöstrand, J.: Puits multiples en limite semi-classique II. Interaction Moléculaire, Symétries, Perturbation. Ann. Inst. Henri Poincaré 42, 127-212 (1985)

[HSj4] Helffer, B., Sjöstrand, J.: Multiple wells in the semi-classical limit III. Interaction through non-resonant wells. Math. Nachr. 124, 263-313 (1985)

[H] Herbst, I.: Dilation analyticity in constant electric field I. The two-body problem. Commun. Math. Phys. 64, 279-298 (1979)

[HiSi] Hislop, P. D., Sigal, I. M.: Semiclassical theory of shape resonances in quantum mechanics. Mem. Am. Math. Soc. 399 (1989)

[HN] Hislop, P. D., Nakamura, S.: Semiclassical resolvent estimates. Ann. Inst. Henri Poincaré 51, 187-198 (1989)

[Hz] Hunziker, W.: Distortion analyticity and molecular resonance curves. Ann. Inst. Henri Poincaré 45, 399-358 (1986)

[K] Klein, M.: On the absence of resonances for Schrödinger operators with nontrapping potentials in the classical limit. Commun. Math. Phys. 106, 485-494 (1986)

[Ka] Kato, T.: Perturbation Theory for Linear Operators. Berlin, Heidelberg, New York: Springer 1980

[N] Nakamura, S.: A note on the absence of resonances for Schrödinger operators. Lett. Math. Phys. 16, 217-233 (1988)

[NN] Nenciu, A., Nenciu, G.: Dynamics of Bloch electrons in external electric fields: I. Bounds for interband transitions and effective Wannier Hamiltonians. J. Phys. A14, 2817-2827 (1981); II. The existence of Stark Wannier ladder resonances. J. Phys. A15, 3313-3328 (1982)

[RS1] Reed, M., Simon, B.: Methods of Modern Mathematical Physics, II. Fourier Analysis. Self-adjointness. New York: Academic Press 1975

[RS2] Reed, M., Simon, B.: Methods of Modern Mathematical Physics, IV. Analysis of operators. New York: Academic Press 1978

[Si1] Sigal, I. M.: Sharp exponential bounds on resonance states and width of resonances. Adv. Appl. Math. 9, 127-166 (1988) 
[Si2] Sigal, I. M.: Complex transformation method and resonances in one-body quantum systems. Ann. Inst. Henri Poincare 41, 103-114 (1984); and Addendum 41, 333 (1984)

[Si3] Sigal, I. M.: Geometric theory of Stark resonances in multielectron systems. Commun. Math. Phys. 119, 287-314 (1988)

[S1] Simon, B.: Semiclassical analysis of low lying eigenvalues I. Non degenerate minima: Asymptotic expansions. Ann. Inst. Henri Poincaré 38, 295-307 (1983)

[S2] Simon, B.: Semiclassical analysis of low lying eigenvalues, II. Tunneling. Ann. Math. 120, 89-118 (1984)

Communicated by B. Simon 